\title{
FINITENESS IN THE MINIMAL MODELS OF SULLIVAN
}

\author{
BY \\ STEPHEN HALPERIN $\left({ }^{l}\right)$
}

\begin{abstract}
Let $X$ be a 1-connected topological space such that the vector spaces $\Pi_{*}(X) \otimes \mathbf{Q}$ and $H^{*}(X ; \mathbf{Q})$ are finite dimensional. Then $H^{*}(X ; \mathbf{Q})$ satisfies Poincare duality. Set $\chi_{\Pi}=\Sigma(-1)^{p} \operatorname{dim} \Pi_{p}(X) \otimes \mathbf{Q}$ and $\chi_{c}=$ $\Sigma(-1)^{p} \operatorname{dim} H^{p}(X ; \mathbf{Q})$. Then $\chi_{\Pi}<0$ and $\chi_{c}>0$. Moreover the conditions: (1) $\chi_{\Pi}=0$, (2) $\chi_{c}>0, H^{*}(X ; \mathbf{Q})$ evenly graded, are equivalent. In this case $H^{*}(X ; \mathrm{Q})$ is a polynomial algebra truncated by a Borel ideal.

Finally, if $X$ is a finite 1-connected C.W. complex, and an $r$-torus acts continuously on $X$ with only finite isotropy, then $\chi_{\Pi}<-r$.
\end{abstract}

1. Introduction. In this paper all vector spaces are defined over a field, $\Gamma$, of characteristic zero. We shall consider positively graded finite dimensional vector spaces $R=\sum_{k \geqslant 0} R^{k}$ ( $R^{k}$ is the subspace of elements of degree $k$ ) with homogeneous bases $x_{1}, \ldots, x_{n}$. The free commutative algebra over $R$ is written $F(R)$ or $F\left(x_{1}, \ldots, x_{n}\right) \cdot\left[F^{l}(R)\right]^{k}$ denotes the subspace spanned by elements of the form $x_{i_{1}} \cdots \cdots x_{i_{1}}$ with $\Sigma_{\nu} \operatorname{deg} x_{i_{i}}=k$. Such elements are called homogeneous of degree $k$.

Write $R=Q \oplus P$ where $Q$ (respectively $P$ ) is the space spanned by the elements of even (respectively odd) degree. Then $F(R)=\bigvee Q \otimes \wedge P$ is the tensor product of the symmetric algebra $\vee Q$ over $Q$ with the exterior algebra $\bigwedge P$ over $P$. We can also write $F(R)=F\left(x_{1}\right) \otimes \cdots \otimes F\left(x_{n}\right)$.

Now suppose $\left(A, d_{A}\right)$ is a graded commutative differential algebra (positively graded, associative, with identity $1 \in A^{\circ}$ ) and suppose $\tau: R \rightarrow A \otimes$ $F(R)$ is a linear map, homogeneous of degree 1 . Then $\tau$ extends to a unique derivation, $d_{\tau}$, of degree 1 in $A \otimes F(R)$ such that $d_{\tau}(a \otimes 1)=0$. Extend $d_{A}$ to $A \otimes F(R)$ by writing $d_{A}(a \otimes z)=d_{A} a \otimes z$.

Definition. $\left(A, d_{A} ; \tau ; x_{1}, \ldots, x_{n}\right)$ will be called a finite tower over $A$ if

$$
\tau\left(x_{1}\right) \in A, \quad \tau\left(x_{i}\right) \in A \otimes F\left(x_{1}, \ldots, x_{i-1}\right) \quad(i \geqslant 2)
$$

and

$$
\left(d_{\tau}+d_{A}\right)^{2}=0
$$

Received by the editors November 4,1975 .

AMS (MOS) subject classifications (1970). Primary 55J15, 55H05; Secondary 57E10.

Key words and phrases. Minimal models, homotopy Euler characteristic, Koszul complex, torus action, finite isotropy.

(') This research is supported by NRC grant A-8047. 
The graded differential algebra $\left(A \otimes F(R), d_{\tau}+d_{A}\right)$ is called the Koszul complex of the tower, and the cohomology algebra $H(A \otimes F(R))$ is called the cohomology of the tower.

If $A=\Gamma$ then $\left(\tau ; x_{1}, \ldots, x_{n}\right)$ will be called simply a finite tower. (In this case $\tau\left(x_{1}\right)=0$.) If $\operatorname{deg} x_{i}>0$ (respectively $>k$ ) for all $i$ then the tower is called connected (respectively $k$-connected).

Let $\left(F(R), d_{\tau}\right)$ be the Koszul complex of a finite tower. The number

$$
\chi_{\Pi}=\sum_{k}(-1)^{k} \operatorname{dim} R^{k}
$$

is called the homotopy Euler characteristic of the tower. If $\operatorname{dim} H\left(F(R), d_{\tau}\right)<$ $\infty$ the tower is called $c$-finite; in this case

$$
\chi_{c}=\sum_{k}(-1)^{k} \operatorname{dim} H^{k}(F(R))
$$

is called the cohomology Euler characteristic. Finally, if

$$
\tau\left(x_{i}\right) \in F^{+}\left(x_{1}, \ldots, x_{i-1}\right) \cdot F^{+}\left(x_{1}, \ldots, x_{i-1}\right), \quad i=2,3, \ldots,
$$

then the tower is called minimal.

Among the principal results of this paper is the following:

THEOREM 1. Let $\left(\tau ; x_{1}, \ldots, x_{n}\right)$ be a connected, finite, c-finite minimal tower. Then $\chi_{\Pi} \leqslant 0$ and $\chi_{c} \geqslant 0$. Moreover, the following conditions are equivalent:

(1) $\chi_{\Pi}=0$.

(2) $\chi_{c}>0$.

(3) $H\left(F\left(x_{1}, \ldots, x_{n}\right)\right)$ is evenly graded.

(In fact we shall show that for each $p, \Sigma_{i \geqslant p}(-1)^{i} \operatorname{dim} R^{i} \leqslant 0$ (Corollary 2 in \$6) where $R^{i}$ is the span of the $x_{j}$ with $\operatorname{deg} x_{j}=i$.)

The proof of Theorem 1 is contained in the next six sections. Then, in $\S 8$, we show that under the hypotheses of Theorem $1, H\left(F\left(x_{1}, \ldots, x_{n}\right)\right)$ satisfies Poincare duality, and that the degree $m$ of the top dimensional cohomology class is given by

$$
m=r-\sum_{i=1}^{n}(-1)^{\operatorname{deg} x_{i}} \operatorname{deg} x_{i},
$$

where $r$ is the number of $x_{i}$ of even degree.

In $\S \S 9$ and 10 , we show that if $m$ is even and $\chi_{\Pi}<0$, then the Poincare inner product in $\Sigma_{j} H^{2 j}\left(F\left(x_{1}, \ldots, x_{n}\right)\right)$ is hyperbolic. Finally in $\S 11$, we show that if $\chi_{\Pi \mathrm{I}}=0$ then $\left(F\left(x_{1}, \ldots, x_{n}\right), d_{\tau}\right)$ is isomorphic with a Koszul complex of the form $(\bigvee Q \otimes \wedge P, d)$ with $d(Q)=0$ and $d(P) \subset \bigvee Q$. In this case $H(\bigvee Q \otimes \wedge P) \cong \bigvee Q / I$, where $I$ is the ideal generated by $d(P)$. 
Now consider a connected topological space $X$ and let $A(X)$ be the graded commutative differential algebra of rational differential forms on the singular complex of $X$ (cf. Sullivan [5, §D]): in particular, $H(A(X)) \cong H^{*}(X ; \mathrm{Q})$ (singular cohomology). There is a commutative connected graded differential algebra $(F(R), d)$ (over $\mathrm{Q}$ ) and a homomorphism $\phi: F(R) \rightarrow A(X)$ of graded differential algebras such that

(1) $\phi$ induces an isomorphism of cohomology.

(2) There is a homogeneous basis $\left\{x_{\alpha}\right\}_{\alpha \in \mathcal{T}}$ of $R$, where $\mathcal{T}$ is well ordered, such that $d x_{\alpha}$ is a polynomial in those $x_{\beta}$ with $\beta<\alpha$ and $\operatorname{deg} x_{\beta} \leqslant \operatorname{deg} x_{\alpha}$.

Moreover, $(F(R), d)$ is determined up to isomorphism by these conditions.

We shall call the spaces $R^{k}$ the pseudo dual rational homotopy spaces of $X$, and denote them by $\Pi_{\psi}^{k}(X)$. If $H^{1}(X ; \mathbf{Q})=0$ and $H^{*}(X ; \mathbf{Q})$ has finite type, then these spaces are finite dimensional. If, in addition, $X$ is simply connected then there are natural isomorphisms $[5, \S Z]\left[\Pi_{k}(X) \otimes Q\right]^{*} \cong \Pi_{\psi}^{k}(X)$.

Write $\Pi_{\psi}^{*}(X)=\Sigma_{k} \Pi_{\psi}^{k}(X)$ and $\Pi_{*}(X)=\Sigma_{k} \Pi_{k}(X)$. Then the remarks above, together with Theorem 1, yield:

THEOREM $1^{\prime}$. Let $X$ be a connected topological space such that $\Pi_{\psi}^{*}(X)$ and $H^{*}(X ; \mathbf{Q})$ are finite dimensional. Then

$$
\sum_{k}(-1)^{k} \operatorname{dim} \Pi_{\psi}^{k}(X) \leqslant 0 \text { and } \sum_{k}(-1)^{k} \operatorname{dim} H^{k}(X ; \mathbf{Q}) \geqslant 0 .
$$

Moreover, the following conditions are equivalent:

(1) $\Sigma_{k}(-1)^{k} \operatorname{dim} \Pi_{\psi}^{k}(X)=0$.

(2) $\Sigma_{k}(-1)^{k} \operatorname{dim} H^{k}(X ; \mathbf{Q})>0$.

(3) $H^{p}(X ; \mathbf{Q})=0, p$ odd.

COROLlaRY 1. If $X$ is simply connected then the theorem remains true if $\Pi_{\psi}^{k}(X)$ is replaced by $\Pi_{k}(X) \otimes \mathbf{Q}$ everywhere in the statement.

Finally, we have the following application to transformation groups (see Remark 3 below):

THEOREM T. Let a compact Lie group $G$ of rank $r$ act on a simply connected finite C.W. complex $X$ with only finite isotropy. Assume that $\Pi_{*}(X) \otimes \mathbf{Q}$ is finite dimensional. Then $\Sigma(-1)^{k} \operatorname{dim} \Pi_{k}(X) \otimes \mathbf{Q} \leqslant-r$.

Proof. According to Allday [1, Theorem 2.1.1, p. 177] this follows from Theorem 1'(1).

REMARKs. 1. The special case of a finite tower over $A$ with $R$ oddly graded and $\tau(R) \subset A$ was first considered by Koszul [4] in 1950. Cartan [2] showed that the cohomology of a homogeneous space can be calculated via a Koszul complex of this form, where, in addition, $A$ is a symmetric algebra and $d_{A}=0$. Cartan also obtains a special case of Theorem 1; indeed the general theorem will be established by reduction to this earlier result. 
The Koszul complex of a minimal connected tower is a nilpotent minimal model as defined by Sullivan [5].

2. Historically, this paper begins with Theorem $\mathrm{T}$ which was conjectured by W. Y. Hsiang in 1969 or earlier. Then in 1971 Allday [1] reduced Hsiang's conjecture to Theorem $1^{\prime}\left(\chi_{\Pi} \leqslant 0\right)$ in the simply connected case. The translation from Theorem 1' (1) to Theorem 1(1) was observed by Sullivan who poses it as question 5 in $[5, \S \mathrm{Q}]$.

3. Theorem $\mathrm{T}$ remains valid for a much wider class of spaces, $X$. In particular it is sufficient to assume that $X$ is connected (but not necessarily simply connected) if we replace $\Pi_{*}(X) \otimes \mathbf{Q}$ by $\Pi_{\psi}^{*}(X)$ everywhere in the statement. Precise statements and details of the proof will appear elsewhere. As a special case of this generalized Theorem $\mathrm{T}$, however, we have

THEOREM H. Let $K \subset G$ be compact Lie groups and suppose a torus, $T$, acts on $G / K$ continuously, with only finite isotropy. Then $\operatorname{dim} T \leqslant \operatorname{rank} G-$ $\operatorname{rank} K$.

When $K=(e)$ this is proved by Allday [1]. If $G / K$ is 1 -connected then Theorem $\mathrm{H}$ follows from the "ungeneralized" Theorem $\mathrm{T}$.

2. Notation. By a graded commutative differential algebra $\left(A, d_{A}\right)$ we mean a positively graded associative algebra $A=\sum_{k \geqslant 0} A^{k}$ with identity $1 \in A^{\circ}$ such that $a b=(-1)^{r s} b a, a \in A^{r}, b \in A^{s}$. Here $d_{A}$ denotes a derivation of degree 1 with $d_{A}^{2}=0$. The cohomology algebra $\operatorname{ker} d_{A} / \operatorname{Im} d_{A}$ is written $H(A)=\Sigma_{k} H^{k}(A)$. A homomorphism $\phi:\left(A, d_{A}\right) \rightarrow\left(B, d_{B}\right)$ of graded differential algebra induces a homomorphism $\phi^{*}: H(A) \rightarrow H(B)$.

The tensor product of graded algebras $A$ and $B$ is given the multiplication defined by $(a \otimes b)\left(a^{\prime} \otimes b^{\prime}\right)=(-1)^{q p^{\prime}} a a^{\prime} \otimes b b^{\prime}, b \in B^{q}, a^{\prime} \in A^{p^{\prime}}$.

The subspace of a vector space spanned by elements $u_{1}, \ldots$ is denoted by $\left(u_{1}, \ldots\right)$. If $U$ and $V$ are subspaces of a vector space $W, U+V$ is the subspace spanned by $U$ and $V$. If $W$ is an algebra, $U \cdot V$ is the subspace spanned by elements of the form $u v, u \in U, v \in V ; U \cdot U$ is written $U^{2}$.

An evenly (respectively oddly) graded space is a space with no ronzero elements of odd (respectively even) degree.

The identity map of any set is denoted by $\iota$.

Let $R=\left(x_{1}, \ldots, x_{n}\right)$ be as in the introduction, and suppose $\left(A, d_{A} ; \tau ; x_{1}, \ldots, x_{n}\right)$ is a tower over $A$ with Koszul complex $(A \otimes F(R), d)$. Then for each $m,\left(A, d_{A} ; \tau ; x_{1}, \ldots, x_{m}\right)$ is a tower over $A$ with Koszul complex the subdifferential algebra $\left(A \otimes F\left(x_{1}, \ldots, x_{m}\right), d\right)$. Write this $\left(B, d_{B}\right)$.

Then $A \otimes F(R)=B \otimes F\left(x_{m+1}, \ldots, x_{n}\right)$ and so we may regard $\tau$ as a linear map $\tau:\left(x_{m+1}, \ldots, x_{n}\right) \rightarrow B \otimes F\left(x_{m+1}, \ldots, x_{n}\right)$. Clearly $\left(B, d_{B} ; \tau ; x_{m+1}, \ldots, x_{n}\right)$ is a tower over $B$ whose Koszul complex coincides 
with the Koszul complex $(A \otimes F(R), d)$.

Next, let $\left(\tau ; x_{1}, \ldots, x_{n}\right)$ be a finite tower and denote by $\left(B, d_{B}\right)$ the subdifferential algebra $F\left(x_{1}, \ldots, x_{m}\right)$ of $(F(R), d)$. Then, as above, $(F(R), d)$ is also the Koszul complex of the tower $\left(B, d_{B} ; \tau ; x_{m+1}, \ldots, x_{n}\right)$ over $B$. The projection $\rho: B \rightarrow \Gamma$ satisfies $\rho \circ d_{B}=0$. Hence by Lemma 1 , below, it determines a tower $\left(\bar{\tau} ; x_{m+1}, \ldots, x_{n}\right)$ with

$$
\bar{\tau}\left(x_{i}\right)=(\rho \otimes \cdot \iota)\left(\tau x_{i}\right) \in F\left(x_{m+1}, \ldots, x_{n}\right), \quad i=m+1, \ldots, n .
$$

The maps

$$
F\left(x_{1}, \ldots, x_{m}\right) \rightarrow F\left(x_{1}, \ldots, x_{n}\right)
$$

and

$$
\rho \otimes \iota: F\left(x_{1}, \ldots, x_{n}\right) \rightarrow F\left(x_{m+1}, \ldots, x_{n}\right)
$$

are homomorphisms of graded differential algebras. They will be called, respectively, a base inclusion and a fibre projection.

Finally, suppose $\left(A, d_{A} ; \tau ; x_{1}, \ldots, x_{n}\right)$ is a tower over $A$. Let $\omega \in S_{n}$ be some permutation such that for each $i, \tau\left(x_{\omega(i)}\right) \in A \otimes F\left(x_{\omega(1)}, \ldots, x_{\omega(i-1)}\right)$. Then $\left(A, d_{A} ; \tau ; x_{\omega(1)}, x_{\omega(2)}, \ldots, x_{\omega(n)}\right)$ is again a tower over $A$; it is called a rearrangement of the original tower, and has the same Koszul complex.

Observe that the following properties of a tower $\left(\tau ; x_{1}, \ldots, x_{n}\right): c-$ finiteness, $k$-connectivity, minimality depend only on the Koszul complex, and so hold for any rearrangement. (In particular, the tower is minimal if and only if $\tau(R) \subset F^{+}(R) \cdot F^{+}(R)$.) If $\left(\tau ; x_{1}, \ldots, x_{n}\right)$ is a minimal connected tower then there is a permutation, $\omega$, such that $\operatorname{deg} x_{\omega(1)} \leqslant \operatorname{deg} x_{\omega(2)} \leqslant \ldots$, and $\left(\tau ; x_{\omega(1)}, \ldots, x_{\omega(n)}\right)$ is again a tower.

Lemma 1. Suppose $\left(A, d_{A} ; \tau ; x_{1}, \ldots, x_{n}\right)$ is a tower, and let $\phi:\left(A, d_{A}\right) \rightarrow$ $\left(B, d_{B}\right)$ be a homomorphism of graded commutative differential algebras. Define $\sigma: R \rightarrow B \otimes F(R)$ by $\sigma\left(x_{i}\right)=(\phi \otimes \imath)\left(\tau x_{i}\right)$.

Then $\left(B, d_{B} ; \sigma ; x_{1}, \ldots, x_{n}\right)$ is a tower over $B$ and $\phi \otimes \imath: A \otimes F(R) \rightarrow B \otimes$ $F(R)$ is a homomorphism of graded differential algebras. Moreover if $\phi^{*}$ : $H(A) \rightarrow H(B)$ is an isomorphism then $(\phi \otimes \iota)^{*}$ is an isomorphism.

Proof. Clearly $\sigma\left(x_{i}\right) \in B \otimes F\left(x_{1}, \ldots, x_{i-1}\right)$. Moreover

$$
d_{\sigma} \circ(\phi \otimes \iota)\left(1 \otimes x_{i}\right)=\sigma\left(x_{i}\right)=(\phi \otimes \iota) \circ d_{\tau}\left(1 \otimes x_{i}\right)
$$

and

$$
d_{\sigma} \circ(\phi \otimes \iota)(a \otimes 1)=d_{\sigma}(\phi a \otimes 1)=0=(\phi \otimes \iota) \circ d_{\tau}(a \otimes 1) .
$$

Since $d_{\sigma} \circ(\phi \otimes \imath)-(\phi \otimes \imath) \circ d_{\tau}$ is a $(\phi \otimes \imath)$-derivation these equations imply that it is zero:

$$
d_{\sigma} \circ(\phi \otimes \iota)=(\phi \otimes \iota) \circ d_{\tau} .
$$

Hence also $\left(d_{\sigma}+d_{B}\right) \circ(\phi \otimes \imath)=(\phi \otimes \imath) \circ\left(d_{\tau}+d_{A}\right)$. 
Now we obtain

$$
\begin{aligned}
\left(d_{\sigma}+d_{B}\right)^{2}\left(1 \otimes x_{i}\right) & =\left(d_{\sigma}+d_{B}\right)^{2}(\phi \otimes \iota)\left(1 \otimes x_{i}\right) \\
& =(\phi \otimes \iota)\left(d_{\tau}+d_{A}\right)^{2}\left(1 \otimes x_{i}\right)=0 .
\end{aligned}
$$

Since $\left(d_{\sigma}+d_{B}\right)^{2}(b \otimes 1)=d_{B}^{2}(b) \otimes 1=0$, it follows that $\left(d_{\sigma}+d_{B}\right)^{2}=0$. Thus $\left(B, d_{B} ; \sigma ; x_{1}, \ldots, x_{n}\right)$ is a tower and $\phi \otimes \iota$ is a homomorphism of graded differential algebras.

Finally, suppose $\phi^{*}$ is an isomorphism. We shall show (by induction on $m$ ) that the restrictions

$$
(\phi \otimes \iota)_{m}: A \otimes F\left(x_{1}, \ldots, x_{m}\right) \rightarrow B \otimes F\left(x_{1}, \ldots, x_{m}\right)
$$

induce isomorphisms of cohomology.

Suppose first that $m=1$. Filter $A \otimes F\left(x_{1}\right)$ and $B \otimes F\left(x_{1}\right)$ by the subspaces

$$
L^{-p}=\sum_{j=0}^{p} A \otimes F^{j}\left(x_{1}\right) \quad \text { and } \quad \hat{L}^{-p}=\sum_{j=0}^{p} B \otimes F^{j}\left(x_{1}\right), \quad p=0,1, \ldots
$$

Then $\phi \otimes \iota$ is filtration preserving, and so it induces a homomorphism $\alpha_{i}$ : $\left(E_{i}, d_{i}\right) \rightarrow\left(\hat{E}_{i}, \hat{d}_{i}\right)$ of spectral sequences. In particular, $\alpha_{1}$ is given by

$$
\alpha_{1}=\phi^{*} \otimes \iota: H(A) \otimes F\left(x_{1}\right) \stackrel{\cong}{\rightarrow} H(B) \otimes F\left(x_{1}\right) .
$$

Thus each $\alpha_{i}(1 \leqslant i<\infty)$ is an isomorphism. Since $E_{i}^{p, q}=0=\hat{E}_{i}^{p, q}$ for $p>0$ we have $E_{\infty}^{p, q}=$ ind $\lim E_{i}^{p, q}$ ( $i$ large). It follows that $\alpha_{\infty}$ is an isomorphism. Hence $(\phi \otimes \iota)^{*}$ induces an isomorphism in the bigraded algebra determined by the filtrations in $H\left(A \otimes F\left(x_{1}\right)\right)$ and $H\left(B \otimes F\left(s_{1}\right)\right)$. This implies that $(\phi \otimes \imath)^{*}$ is an isomorphism.

Finally, assume by induction that $(\phi \otimes \imath)_{m-1}^{*}$ is an isomorphism. Write $(\phi \otimes \iota)_{m-1}=\psi, A \otimes F\left(x_{1}, \ldots, x_{m-1}\right)=A^{\prime}, B \otimes F\left(x_{1}, \ldots, x_{m-1}\right)=B^{\prime}$. Apply the argument above to

$$
\phi_{m} \otimes \iota=\psi \otimes \iota: A^{\prime} \otimes F\left(x_{m}\right) \rightarrow B^{\prime} \otimes F\left(x_{m}\right)
$$

to obtain that $\left(\phi_{m} \otimes \iota\right)^{*}$ is an isomorphism. Q.E.D.

EXAMPLE. Let $\left(A, d_{A} ; \tau ; x_{1}\right)$ be a tower with deg $x_{1}$ odd. Its Koszul complex is given by $\left(A \otimes \wedge x_{1}, d\right)$, where

$$
d\left(a \otimes x_{1}+b \otimes 1\right)=d_{A} a \otimes x_{1}+\left((-1)^{\operatorname{deg} a} a \cdot \tau\left(x_{1}\right)+d_{A} b\right) \otimes 1 .
$$

In particular $\tau\left(x_{1}\right)$ is a cocycle representing a class $\alpha \in H(A)$.

A short exact sequence $0 \rightarrow A \rightarrow{ }^{\phi} A \otimes \wedge x_{1} \rightarrow{ }^{\Psi} A \rightarrow 0$ is given by $\phi a=a$ $\otimes 1, \Psi\left(a \otimes x_{1}+b \otimes 1\right)=a$. The ensuing long exact (Gysin) sequence in cohomology has connecting homomorphism $\partial: H(A) \rightarrow H(A)$ given by $\partial \beta=$ $\alpha \cdot \beta$. This sequence yields the short exact sequence 


$$
0 \rightarrow \operatorname{Coker} \partial \stackrel{\bar{\phi}^{*}}{\rightarrow} H\left(A \otimes \wedge x_{1}\right) \stackrel{\Psi^{*}}{\rightarrow} \text { Ker } \partial \rightarrow 0 .
$$

(Cf. [3, Chapter III] for details.)

3. Pure towers. Let $\left(\sigma ; x_{1}, \ldots, x_{n}\right)$ be a finite tower. As in $\S 1$ write $R=\left(x_{1}, \ldots, x_{n}\right)=Q \oplus P$ where $Q$ is evenly graded and $P$ is oddly graded. The tower will be called pure if $\sigma(P) \subset \vee Q$ and $\sigma(Q)=0$. Koszul complexes of pure towers were studied by Koszul [4] and H. Cartan [2]; we recall here some of their results.

Let $(\bigvee Q \otimes \wedge P, d)$ be the Koszul complex of a pure tower $\left(\sigma ; x_{1}, \ldots\right.$, $\left.x_{n}\right)$. Then $d: \bigvee Q \otimes \wedge^{i} P \rightarrow \bigvee Q \otimes \wedge^{i-1} P$, and thus the gradation $\bigvee Q \otimes$ $\wedge P=\Sigma_{k} \vee Q \otimes \wedge^{k} P$ leads to a gradation of $H(\bigvee Q \otimes \wedge P)$, written $H(\bigvee Q \otimes \wedge P)=\Sigma_{k} H_{k}(\bigvee Q \otimes \wedge P)$. Let $\bigvee Q \circ P$ be the ideal in $\bigvee Q$ generated by $\sigma(P)$; then the inclusion $l: \bigvee Q \rightarrow \bigvee Q \otimes \wedge P$ induces an isomorphism $l^{*}: \bigvee Q / \bigvee Q \circ P \rightarrow \approx H_{0}(\bigvee Q \otimes \wedge P)$.

If $P_{1} \subset P$ is any graded subspace then $\bigvee Q \otimes \wedge P_{1}$ is a subdifferential algebra of $\bigvee Q \otimes \wedge P$.

Lemma 2. If $H_{k}\left(\bigvee Q \otimes \wedge P_{1}\right) \neq 0$ then $H_{k}(\bigvee Q \otimes \wedge P) \neq 0$.

Proof. By considering a sequence of spaces $P_{1} \subset P_{2} \subset \cdots \subset P_{m}=P$ we can reduce to the case $P=P_{1} \oplus(x)$. Set $\left(A, d_{A}\right)=\left(\bigvee Q \otimes \wedge P_{1}, d\right)$; then $\bigvee Q \otimes \wedge P=A \otimes \wedge x$ is the Koszul complex of the tower $\left(A, d_{A} ; \sigma ; x\right)$.

Now apply the example of $\$ 2$ to obtain a Gysin sequence in which the connecting homomorphism $\partial: H(A) \rightarrow H(A)$ is multiplication by the class $\alpha \in H(A)$ represented by $\sigma(x)$. Since $\sigma(x) \in \bigvee Q$ it follows that for some $p>0, \alpha \in H_{0}^{p}\left(\bigvee Q \otimes \wedge P_{1}\right)$. This implies that $\partial$ restricts to linear maps $\partial_{i}$ : $H_{i}\left(\bigvee Q \otimes \wedge P_{1}\right) \rightarrow H_{i}\left(\bigvee Q \otimes \wedge P_{1}\right)$ of positive degree. In particular, since $H_{k}\left(\bigvee Q \otimes \wedge P_{1}\right) \neq 0$, then Coker $\partial_{k} \neq 0$.

Finally note that the inclusion Coker $\partial \rightarrow H(A \otimes \wedge x)$ of the example in $\S 2$ is the direct sum of inclusions Coker $\partial_{i} \rightarrow H_{i}(\vee Q \otimes \wedge P)$. Thus since Coker $\partial_{k} \neq 0$ we have $H_{k}(\bigvee Q \otimes \wedge P) \neq 0$. Q.E.D.

The following is due to Cartan [2]. A detailed proof is given in [3, Chapter 2].

TheOREM 2. Let $(\bigvee Q \otimes \wedge P, d)$ be the Koszul complex of a connected pure tower such that $\operatorname{dim} H(\bigvee Q \otimes \wedge P)<\infty$. Then $H(\bigvee Q \otimes \wedge P)$ has nonnegative Euler characteristic $\chi$. Moreover $\operatorname{dim} P-\operatorname{dim} Q$ is the nonnegative integer $k$ with the property

$$
H_{k}(\bigvee Q \otimes \wedge P) \neq 0, \quad H_{k+p}(\bigvee Q \otimes \wedge P)=0, \quad p \geqslant 1
$$

Finally, the following conditions are equivalent:

(i) $\operatorname{dim} P=\operatorname{dim} Q$.

(ii) $\chi>0$. 
(iii) $H(\bigvee Q \otimes \wedge P)$ is evenly graded.

(iv) $H(\bigvee Q \otimes \wedge P)=H_{0}(\bigvee Q \otimes \wedge P)$.

Remark. $\operatorname{dim} H(\bigvee Q \otimes \wedge P)<\infty$ if and only if $\operatorname{dim} \vee Q \wedge \vee \circ P<$ $\infty$. In fact note that ker $d$ is a $\vee Q$-submodule of the finitely generated $\bigvee Q$-module $\bigvee Q \otimes \wedge P$. Because $\vee Q$ is noetherian ker $d$ is finitely generated. Thus $H(\vee Q \otimes \wedge P)$ is a finitely generated $\vee Q$ module.

This implies (clearly) that $H(\bigvee Q \otimes \wedge P)$ is a finitely generated module over $H_{0}(\bigvee Q \otimes \wedge P)$. Thus $\operatorname{dim} H(\bigvee Q \otimes \wedge P)<\infty$ if and only if $\operatorname{dim} H_{0}(\bigvee Q \otimes \wedge P)<\infty$; i.e., if and only if $\operatorname{dim} \bigvee Q / \vee Q \circ P<\infty$.

4. The $S$-spectral sequence. As in $\S 1$ let $R=\left(x_{1}, \ldots, x_{n}\right)$. Assume deg $x_{i}$ $>0, i=1, \ldots, n$. Let $S$ be a subspace spanned by some of the $x_{i}$ and let $T$ be the subspace spanned by the remaining $x_{j}$. (Then $R=T \oplus S$.)

Now suppose $\left(A, d_{A} ; \tau ; x_{1}, \ldots, x_{n}\right)$ is a tower over $A$. Then $A \otimes F(R)=$ $A \otimes F(T) \otimes F(S)$ and so a bigradation of $A \otimes F(R)$ is given by

$$
[A \otimes F(R)]^{p, q}=\left[A \otimes F(T) \otimes F^{-q}(S)\right]^{p+q} \text {. }
$$

Write $\left(A \otimes F(R), d_{\tau}+d_{A}\right)=\left(C, d_{c}\right): C=\Sigma_{p, q} C^{p, q}$.

Clearly $C^{p, q} \cdot C^{r, s} \subset C^{p+r, q+s}$ and so $C$ is filtered by the ideals $I^{p}=$ $\sum_{j>p} C^{j, *}$. (Note $C^{p, q}=0$ if $p<0$.)

Now let $\sigma: R \rightarrow A \otimes F(T)$ be the unique linear map such that $\sigma(x)=0$, $x \in T$ and $\sigma(x)-\tau(x) \in A \otimes F(T) \otimes F^{+}(S), x \in S$. Extend $\sigma$ to a derivation $d_{\sigma}$ in $C$ such that $d_{\sigma}(A)=0$. Clearly $d_{\sigma}^{2}=0$.

LEMMA 3. (i) $d_{\sigma}$ is homogeneous of bidegree $(0,1)$.

(ii) $d_{c}-d_{\sigma}: I^{p} \rightarrow I^{p+1}$.

\section{Proof. Clear.}

The lemma shows that the $I^{p}$ filter the graded differential algebra $\left(C, d_{c}\right)$, and that the first term of the resulting spectral sequence (of graded differential algebras) is given by

$$
\left(E_{0}, d_{0}\right) \cong\left(C, d_{\sigma}\right) \text {. }
$$

Moreover, because the elements in $F^{q}(S)$ have degree at least $q$, it follows that $C^{p, q}=0$ unless $0 \leqslant-2 q \leqslant p$. This implies that the spectral sequence converges to $H\left(C, d_{c}\right)$. This spectral sequence will be called the $S$-spectral sequence.

In particular, if $P$ denotes the subspace of $R$ of elements of odd degree then the $P$-spectral sequence will be called the odd spectral sequence.

5. The odd spectral sequence of a tower. Let $R=\left(x_{1}, \ldots, x_{n}\right)$ and suppose $\left(\tau ; x_{1}, \ldots, x_{n}\right)$ is a connected finite tower. As usual write $F(R)=\bigvee Q \otimes$ $\wedge P$. 
Let $\sigma: R \rightarrow \bigvee Q$ be the linear map defined by $\sigma(Q)=0$ and $\sigma(x)-\tau(x)$ $\in \bigvee Q \otimes \wedge^{+} P, x \in P$. Then $\left(\sigma ; x_{1}, \ldots, x_{n}\right)$ is a pure tower, called the associated pure tower for $\left(\tau ; x_{1}, \ldots, x_{n}\right)$.

Observe as well that $\tau(Q) \subset F(R)^{\text {odd }} \subset \vee Q \otimes \wedge^{+} P$. It follows that

$$
d_{\tau}-d_{\sigma}: \bigvee Q \otimes \wedge P \rightarrow \bigvee Q \otimes \wedge^{+} P
$$

If $\left(E_{i}, d_{i}\right)$ is the odd spectral sequence for the original tower then

$$
\left(E_{0}, d_{0}\right) \cong\left(\bigvee Q \otimes \wedge P, d_{\sigma}\right)
$$

(cf. formula (4), §4). This isomorphism restricts to isomorphisms $E_{0}^{p, q} \cong(\bigvee Q$ $\left.\otimes \wedge^{-q} P\right)^{p+q}$. Thus there is an algebra isomorphism

$$
E_{1} \cong H\left(\vee Q \otimes \wedge P, d_{\sigma}\right)
$$

which restricts to isomorphisms

$$
E_{1}^{p, q} \cong H_{-q}^{p+q}\left(\bigvee Q \otimes \wedge P, d_{\sigma}\right)
$$

Now we show that $d_{1}=0$, so that $E_{2} \cong E_{1}$. In fact by (5), $\left(d_{\tau}-d_{\sigma}\right)(Q) \subset$ $\vee Q \otimes \wedge^{+} P$ while

$$
\begin{aligned}
\left(d_{\tau}-d_{\sigma}\right)(P) & \subset\left(\bigvee Q \otimes \wedge^{+} P\right) \cap\left(\sum_{r \text { even }}\left(\bigvee Q \otimes \wedge^{r}\right)\right. \\
& \subset \sum_{j>2} \bigvee Q \otimes \wedge^{j} P
\end{aligned}
$$

Thus

$$
\left(d_{\tau}-d_{\sigma}\right):\left(\bigvee Q \otimes \wedge^{-q} P\right)^{p+q} \rightarrow \sum_{j>1}\left(\bigvee Q \otimes \wedge^{-q+j} P\right)^{p+q+1}
$$

It follows that $d_{\tau}-d_{\sigma}: I^{p} \rightarrow I^{p+2}$ (the $I^{p}$ are the ideals defining the spectral sequence) and hence the differential $d_{1}=0$.

Similarly, it follows at once from the definition that

$$
I^{p} \cap F(R)^{r}=\sum_{k>p-r}\left(\bigvee Q \otimes \wedge^{k} P\right)^{r}
$$

Thus if $J=\bigvee Q \otimes \bigwedge^{+} P$ and $J_{k}=J \cdots \cdots J$ ( $k$ factors $)$, then $I^{p} \cap F(R)^{r}$ $=\sum_{k>p-r} J_{k} \cap F(R)^{r}$.

Now suppose $F\left(R^{\prime}\right)=\bigvee Q^{\prime} \otimes \wedge P^{\prime}$ is the Koszul complex of a second finite tower and assume $\phi: F(R) \rightarrow F\left(R^{\prime}\right)$ is a homomorphism of graded differential algebras. Since $J$ and $J^{\prime}$ are the ideals generated by elements of odd degree, $\phi\left(J_{k}\right) \subset J_{k}^{\prime}, k=1,2, \ldots$

Now the formula above for $I^{p}$ shows that $\phi$ is filtration preserving. Hence it induces a homomorphism of spectral sequences. In particular, if $\phi$ is an isomorphism then $\phi^{-1}$ is also filtration preserving and so $\phi$ and $\phi^{-1}$ induce inverse isomorphisms of the odd spectral sequences. 
6. Proof of Theorem 1. Recall from $\S 2$ that a tower $\left(\tau ; x_{1}, \ldots, x_{n}\right)$ determines towers $\left(\bar{\tau} ; x_{p}, \ldots, x_{n}\right)$. Since $\bar{\tau}\left(x_{p}\right)=0, x_{p}$ is a cocycle in $\left(F\left(x_{p}, \ldots, x_{n}\right)\right)$. Let $\left[x_{p}\right] \in H\left(F\left(x_{p}, \ldots, x_{n}\right), d_{\bar{\tau}}\right)$ be the class represented by $x_{p}$.

Proposition 1. Let $\left(\tau ; x_{1}, \ldots, x_{n}\right)$ be a connected, finite, minimal tower. Write $R=\left(x_{1}, \ldots, x_{n}\right), F(R)=\bigvee Q \otimes \wedge P$. Suppose $\left(E_{i}, d_{i}\right)$ denotes the odd spectral sequence and $\left(\sigma ; x_{1}, \ldots, x_{n}\right)$ is the associated pure tower. Then the following are equivalent:

(1) The tower is c-finite: $\operatorname{dim} H\left(\bigvee Q \otimes \wedge P, d_{\tau}\right)<\infty$.

(2) For each $p$ the class $\left[x_{p}\right] \in H\left(F\left(x_{p}, \ldots, x_{n}\right), d_{\bar{\tau}}\right)$ is nilpotent: $\left[x_{p}\right]^{k}=0$, some $k$.

(3) $\operatorname{dim} H\left(\bigvee Q \otimes \wedge P, d_{\mathbf{o}}\right)<\infty$.

(4) $\operatorname{dim} E_{1}<\infty$.

Proof. (1) $\Rightarrow(2)$. This is deferred until $\$ 7$ (Lemma 5).

$(2) \Rightarrow(3)$. Denote by $Q_{p}$ the subspace of $Q$ spanned by the $x_{i}$ of even degree with $i \leqslant p$, and set $Q_{0}=0$. We show first by induction on $p$ that the elements of $Q_{p}$ represent nilpotent classes in $H\left(\bigvee Q \otimes \wedge P, d_{\sigma}\right)$.

This is certainly true for $p=0$. Suppose it is true for $p-1$. If $x_{p}$ has odd degree then $Q_{p}=Q_{p-1}$ and our claim is true for $p$. If $x_{p}$ has even degree our hypothesis shows that for some $u, v_{i} \in \vee Q \otimes \wedge P$ and some $k \geqslant 1$ :

$$
x_{p}^{k}=d_{\tau} u-\sum_{i=1}^{p-1} x_{i} \cdot v_{i} \text {. }
$$

Hence $d_{\tau} u-x_{p}^{k} \in Q_{p-1} \cdot \bigvee Q+\bigvee Q \otimes \wedge^{+} P$. Thus formula (5) yields

$$
d_{o} u-x_{p}^{k} \in Q_{p-1} \cdot \bigvee Q+\bigvee Q \otimes \Lambda^{+} P \text {. }
$$

Now write $u=\sum u_{i}, u_{i} \in \bigvee Q \otimes \wedge^{i} P$. Since $d_{\mathrm{o}}: \bigvee Q \otimes \wedge^{i} P \rightarrow \bigvee Q \otimes$ $\wedge^{i-1} P$, it follows from (9) that

$$
d_{\sigma} u_{1}-x_{p}^{k} \in Q_{p-1} \cdot \bigvee Q
$$

Since the elements of $\bigvee Q$ are $d_{\sigma}$-cocycles and the elements of $Q_{p-1}$ represent nilpotent classes in $H\left(\bigvee Q \otimes \wedge P, d_{\sigma}\right)$, it follows that the elements of $Q_{p-1} \cdot \bigvee Q$ represent nilpotent classes. Hence the equation above implies that $x_{p}$ represents a nilpotent class. The induction is now closed.

We have now shown that the elements in $Q$ represent nilpotent classes in $H\left(\bigvee Q \otimes \wedge P, d_{\sigma}\right)$. This implies that $\bigvee Q$ has finite dimensional image in $H\left(\bigvee Q \otimes \wedge P, d_{\mathrm{o}}\right)$. The remark in $\$ 3$ now implies that

$$
\operatorname{dim} H\left(\bigvee Q \otimes \wedge P, d_{\sigma}\right)<\infty \text {. }
$$

(3) $\Rightarrow(4)$. Apply formula (7).

(4) $\Rightarrow(1)$. Recall that the spectral sequence converges to 


$$
H\left(\bigvee Q \otimes \wedge P, d_{\tau}\right) \text {. Q.E.D. }
$$

COROLlary. If $\left(\tau ; x_{1}, \ldots, x_{n}\right)$ is a connected, finite, c-finite, minimal tower, then for each $p$ the tower $\left(\bar{\tau} ; x_{p}, \ldots, x_{n}\right)$ is also $c$-finite.

THEOREM 1. Let $\left(\tau ; x_{1}, \ldots, x_{n}\right)$ be a connected, finite, $c$-finite, minimal tower. Then $\chi_{\Pi} \leqslant 0$ and $\chi_{c} \geqslant 0$. Moreover, the following conditions are equivalent.

(1) $\chi_{\Pi}=0$.

(2) $\chi_{c}>0$.

(3) $H\left(F\left(x_{1}, \ldots, x_{n}\right), d_{\tau}\right)$ is evenly graded.

Proof. We adopt the notation of Proposition 1. Then according to Proposition $1, H\left(\bigvee Q \otimes \wedge P, d_{\sigma}\right)$ has finite dimension. Denote its Euler characteristic by $\chi$. Since $H\left(\bigvee Q \otimes \wedge P, d_{\sigma}\right) \cong E_{1}$ and since $\left(E_{i}, d_{i}\right)$ converges to $H\left(\bigvee Q \otimes \wedge P, d_{\tau}\right)$ it follows that $\chi=\chi_{c}$.

Moreover, since $H\left(\bigvee Q \otimes \wedge P, d_{\sigma}\right)$ has finite dimension we can apply Theorem $2, \S 3$ to obtain $\chi_{\Pi}=\operatorname{dim} Q-\operatorname{dim} P \leqslant 0$ and $\chi_{c}=\chi \geqslant 0$.

The equivalence of conditions (i), (ii) and (iii) in Theorem 2 implies that conditions (1) and (2) are equivalent, and hold if and only if

$$
H\left(\bigvee Q \otimes \wedge P, d_{\sigma}\right)
$$

is evenly graded. But in this case $E_{1}$ is evenly graded and so the odd spectral sequence collapses at the $E_{1}$-term. In particular, $H\left(\bigvee Q \otimes \wedge P, d_{\tau}\right)$ is evenly graded. Thus $(1) \Leftrightarrow(2) \Rightarrow(3)$. But clearly (3) $\Rightarrow(2)$. Q.E.D.

COROLLARY 1. Let $\left(E_{i}, d_{i}\right)$ be the odd spectral sequence of a connected, finite, $c$-finite minimal tower. Then for $i>1: E_{i}^{p, q}=0, q<\chi_{\Pi}$.

Proof. By formula (8), $E_{1}^{p, q} \cong H_{-q}^{p+q}\left(\bigvee Q \otimes \wedge P, d_{\sigma}\right)$. If $q<\chi_{\Pi}$ then $H_{-q}\left(\bigvee Q \otimes \wedge P, d_{\sigma}\right)=0$ by formula (3), Theorem 2. Thus $E_{1}^{p, q}=0, q<$ $\chi_{\Pi}$ and so $E_{i}^{p, q}=0, q<\chi_{\Pi}$. Q.E.D.

COROLlaRY 2. Let $\left(\tau ; x_{1}, \ldots, x_{n}\right)$ be a connected, finite, c-finite minimal tower. Then for each $p$,

$$
\sum_{i=p}^{n}(-1)^{\operatorname{deg} x_{i}} \leqslant 0
$$

In particular, if $R=\left(x_{1}, \ldots, x_{n}\right)$ then for each $p$,

$$
\sum_{i>p}(-1)^{i} \operatorname{dim} R^{i} \leqslant 0 \text {. }
$$

Proof. In view of the corollary to Proposition 1 we may apply Theorem 1 to the tower $\left(\bar{\tau} ; x_{p}, \ldots, x_{n}\right)$ to obtain (10). Next note (cf. \$2) that we may 
rearrange the $x_{i}$ so that $\operatorname{deg} x_{\omega(1)} \leqslant \operatorname{deg} x_{\omega(2)} \leqslant \ldots$ Now (11) is a special case of (10) (with $x_{i}$ replaced by $x_{\omega(i)}$ ). Q.E.D.

Corollary 3. Let $X$ be a connected topological space such that $H^{*}(X ; \mathbf{Q})$ and $\Pi_{\psi}^{*}(X)$ are finite dimensional. Then for each $p, \Sigma_{i \geqslant p}(-1)^{i} \operatorname{dim} \Pi_{\psi}^{i}(X) \leqslant$ 0 . If $X$ is simply connected then for each $p, \Sigma_{i>p}(-1)^{i} \operatorname{dim} \Pi_{i}(X) \otimes \mathbf{Q} \leqslant 0$.

Proof. This follows from Corollary 2 in the same way Theorem 1' followed from Theorem 1 (cf. §1). Q.E.D.

COROLlary 4. The odd spectral sequence for a connected, finite, c-finite, minimal tower with $\chi_{\Pi}=0$ collapses at the $E_{1}$-term.

Proposition 2 below and its proof are due to C. Allday (private communication). It is a special case of his conjecture $* *$ in [1]; the general case remains open.

Let $A=\Sigma_{k \geq 0} A^{k}$ be a graded vector space of finite type. Its Poincaré series is the formal series $f_{A}(t)=\Sigma_{k} \operatorname{dim} A^{k} t^{k}$. Following Hsiang set

$$
\rho_{0}(A)=\inf \left\{\alpha \in \mathbf{R} \mid(1-t)^{\alpha} f_{A}(t) \rightarrow 0 \text { as } t \rightarrow 1-\right\} .
$$

If $g=\Sigma a_{k} t^{k}$ and $h=\Sigma b_{k} t^{k}$ are two formal series with integer coefficients we write $g \leqslant h$ to mean $a_{k} \leqslant b_{k}$, each $k$.

Proposition 2. Suppose $\left(F(R), d_{\tau}\right)$ is the Koszul complex of a connected, finite, minimal tower $\left(\tau ; x_{1}, \ldots, x_{n}\right)$ with homotopy Euler characteristic $\chi_{\Pi}$. Assume $H(F(R))$ is finitely generated as an algebra over $\Gamma$. Then $\chi_{\Pi} \leqslant$ $\rho_{0}(H(F(R)))$.

REMARK. As will appear in the proof, $\rho_{0}(H(F(R)))$ is the Krull dimension of the commutative algebra $\Sigma_{k} H^{2 k}(F(R))$.

Proof. Denote the commutative subalgebra $\Sigma_{k} H^{2 k}(F(R))$ by $A$. Using the argument of $\left[7\right.$, p. 201] we construct a sequence $z_{0}, \ldots, z_{l}$ of homogeneous elements in $A^{+}$as follows: assume $z_{0}, \ldots, z_{i}$ are constructed with $z_{0}=0$, and generate an ideal $Z_{i}$ with isolated prime ideals $J_{1}, \ldots, J_{k}$ (cf. [6, p. 211]). These are necessarily graded, and hence in $A^{+}$. Thus either $k=1$ and $J_{1}=A^{+}$or there is a homogeneous element $z_{i+1}$ in $A^{+}$such that $z_{i+1} \notin$ $\cup_{\lambda} J_{\lambda}$.

The sequence $Z_{1}, Z_{2}, \ldots$ terminates at some $Z_{l}$ because $A$ is noetherian; in particular, $A^{+}$is the unique prime ideal for $Z_{l}$ and so $A / Z_{l}$ has finite dimension.

Choose a sequence $K_{l} \supset \cdots \supset K_{1} \supset K_{0}$ with $K_{i}$ an isolated prime ideal for $Z_{i}$; then $z_{i} \notin K_{i-1}, i \geqslant 1$. Thus an easy induction on $l-i$ shows that the obvious homomorphism $\bigvee\left(z_{i+1}, \ldots, z_{l}\right) \rightarrow A / Z_{i}$ is injective. In particular we have an inclusion $\bigvee\left(z_{1}, \ldots, z_{l}\right) \rightarrow A$. On the other hand, if $F$ is a (finite dimensional) graded space such that $F \oplus Z_{l}=A$, then the obvious map $\bigvee\left(z_{1}, \ldots, z_{l}\right) \otimes F \rightarrow A$ is surjective. It follows that (denoting $\bigvee\left(z_{1}, \ldots, z_{l}\right)$ 
by $B$ ) that $f_{B} \leqslant f_{A} \leqslant f_{B} f_{F}$, whence $\rho_{0}(A)=\rho_{0}(B)=l$.

Moreover, if $S \subset H^{\text {odd }}(F(R))$ is a finite dimensional graded subspace, which, together with $A$ generates $H(F(R))$, then $f_{A} \leqslant f_{H(F(R))} \leqslant f_{A} \cdot f_{\wedge s}$. Hence $\rho_{0}(H(F(R)))=\rho_{0}(A)=l$.

On the other hand, let $y_{1}, \ldots, y_{l}$ be cocycles representing $z_{1}, \ldots, z_{l}$ and let $U=\left(u_{1}, \ldots, u_{l}\right)$ be a graded space with $\operatorname{deg} u_{i}=\operatorname{deg} z_{i}-1$. Define Koszul complexes $(F(R) \otimes \wedge U, d)$ and $(H(F(R)) \otimes \wedge U, \bar{d})$ by

$$
d(\Phi \otimes 1)=d_{\tau} \Phi \otimes 1, \quad d\left(1 \otimes u_{i}\right)=y_{i} \otimes 1, \quad \bar{d}\left(1 \otimes u_{i}\right)=z_{i} \otimes 1 .
$$

According to [4] there is a spectral sequence converging to

$$
H(F(R) \otimes \wedge U, d)
$$

with $E_{2}$-term $H(H(F(R)) \otimes \wedge U, \bar{d})$. (See [3, Chapter III] for details.) (This spectral sequence, introduced by Koszul, is a special case of the EilenbergMoore spectral sequence.) Since $A / Z_{l}$ has finite dimension, the argument in the remark of $\S 3$ shows that, so does $H(H(F(R)) \otimes \wedge U)$.

It follows that $H(F(R) \otimes \wedge U)$ has finite dimension. Its homotopy Euler characteristic is given by $\chi_{\Pi}-l\left(\chi_{\Pi}\right.$ the homotopy Euler characteristic of $F(R))$. Now apply Theorem 1 to get $\chi_{\Pi}-l \leqslant 0$; i.e. $\chi_{\Pi} \leqslant l=\rho_{0}(H(F(R)))$. Q.E.D.

COROLlaRY. Let $X$ be a connected topological space with $\operatorname{dim} \Pi_{\psi}^{*}(X)<\infty$ and $H^{*}(X ; \mathbf{Q})$ finitely generated. Set $\chi_{\Pi}(X)=\Sigma(-1)^{k} \operatorname{dim} \Pi_{\psi}^{k}(X)$ and $\rho_{0}(X)$ $=\rho_{0}\left(H^{*}(X ; \mathbf{Q})\right)$. Then $\chi_{\Pi}(X) \leqslant \rho_{0}(X)$.

\section{Two lemmas.}

Lemma 4. Suppose $\left(\tau ; x_{1}, \ldots, x_{n}\right)$ is a connected, finite, minimal tower. Then there is a tower $\left(\sigma ; x_{1}, \ldots, x_{n}, y_{1}, \ldots, y_{n}\right)$ with $\operatorname{deg} y_{i}=\operatorname{deg} x_{i}-1$ and with the following properties for each $i(1 \leqslant i \leqslant n)$ :

(i) $\sigma\left(x_{i}\right)=\tau\left(x_{i}\right)$.

(ii) $\sigma\left(y_{i}\right)-x_{i} \in\left(x_{1}, \ldots, x_{i-1}\right) \cdot F\left(x_{1}, \ldots, x_{i-1}, y_{1}, \ldots, y_{i-1}\right)$.

(iii) $H\left(F^{+}\left(x_{1}, \ldots, x_{i}, y_{1}, \ldots, y_{i}\right), d_{\sigma}\right)=0$. (Note that (ii) implies that $F\left(x_{1}, \ldots, x_{i}, y_{1}, \ldots, y_{i}\right)$ is stable under $d_{\sigma}$.)

(iv) If $i<n$ then for some $w \in\left(x_{1}, \ldots, x_{i}\right) \cdot F\left(x_{1}, \ldots, x_{i}, y_{1}, \ldots, y_{i}\right)$, $d_{\sigma}\left(x_{i+1}-w\right)=0$.

Proof. We use induction on $p$ to define elements

$$
\sigma\left(y_{p}\right) \in F\left(x_{1}, \ldots, x_{p}, y_{1}, \ldots, y_{p}\right)
$$

so that conditions (i)-(iv) hold for $i \leqslant p$.

If $p=1$ set $\sigma\left(y_{1}\right)=x_{1}$. Since $\tau\left(x_{1}\right)=0$ it follows that $\left(\sigma ; x_{1}, y_{1}\right)$ and $\left(\sigma ; x_{1}, \ldots, x_{n}, y_{1}\right)$ are towers. Condition (ii) is obvious, while (iii) asserts that $H\left(F^{+}\left(x_{1}, y_{1}\right)\right)=0$; this is a simple and classical calculation. (If $\operatorname{deg} x_{1}$ is odd 
it is essential that $\Gamma$ have characteristic 0 !.)

Finally, since the original tower was minimal, for some $a \in F^{+}\left(x_{1}\right), \tau\left(x_{2}\right)$ $=a x_{1}$. Set $w=(-1)^{\operatorname{deg} a} a y_{1}$. Then in $F\left(x_{1}, x_{2}, y_{1}\right), d_{\sigma}\left(x_{2}-w\right)=a x_{1}-a x_{1}$ $=0$.

Suppose now that $\sigma\left(y_{j}\right)$ is constructed for $j<p$ so that (i)-(iv) hold for $j<p$. Then $\left(\sigma ; x_{1}, \ldots, x_{p}, y_{1}, \ldots, y_{p-1}\right)$ is a tower, and by (iv) there is an element $w \in\left(x_{1}, \ldots, x_{p-1}\right) \cdot F\left(x_{1}, \ldots, x_{p-1}, y_{1}, \ldots, y_{p-1}\right)$ such that $d_{o}\left(x_{p}\right.$ $-w)=0$. Set $\sigma\left(y_{p}\right)=x_{p}-w$. Then $d_{\sigma}^{2}\left(y_{p}\right)=d_{\sigma}\left(x_{p}-w\right)=0$ and so $(\sigma$; $\left.x_{1}, \ldots, x_{p}, y_{1}, \ldots, y_{p}\right)$ is a tower. Hence so is $\left(\sigma ; x_{1}, \ldots, x_{n}, y_{1}, \ldots, y_{p}\right)$.

Moreover (ii) (for $i=p$ ) is immediate from the definition. To check (iii) write $\left(F\left(x_{1}, \ldots, x_{p-1}, y_{1}, \ldots, y_{p-1}\right), d_{\sigma}\right)=\left(A, d_{A}\right)$. Then

$$
\left(F\left(x_{1}, \ldots, x_{p}, y_{1}, \ldots, y_{p}\right), d_{\sigma}\right)
$$

is the Koszul complex of the tower $\left(A, d_{A} ; \sigma, x_{p}, y_{p}\right)$ over $\left(A, d_{A}\right)$.

Let $\rho: A \rightarrow \Gamma$ and $\rho \otimes \iota: A \otimes F\left(x_{p}, y_{p}\right) \rightarrow F\left(x_{p}, y_{p}\right)$ be the projections. By (i) and (ii) (for $i=p)(\rho \otimes \imath)\left(\sigma x_{p}\right)=0$ and $(\rho \otimes \imath)\left(\sigma y_{p}\right)=x_{p}$. Thus if we define $\left(\bar{\sigma} ; x_{p}, y_{p}\right)$ by $\bar{\sigma}\left(y_{p}\right)=x_{p}, \bar{\sigma}\left(x_{p}\right)=0$, then $(\rho \otimes \imath) \circ d_{\sigma}=d_{\bar{\sigma}} \circ(\rho \otimes \imath)$.

By our induction hypothesis (iii) (for $i=p-1$ ), $\rho^{*}$ is an isomorphism. Hence (cf. Lemma $1, \S 2)(\rho \otimes \imath)^{*}$ is an isomorphism. Thus

$$
H\left(F^{+}\left(x_{1}, \ldots, x_{p}, y_{1}, \ldots, y_{p}\right), d_{\sigma}\right) \cong H\left(F^{+}\left(x_{p}, y_{p}\right), d_{\bar{\sigma}}\right)=0 .
$$

It remains to prove (iv). Since $\tau\left(x_{p+1}\right)$ is a cocycle in $F\left(x_{1}, \ldots, x_{p}\right)$, it is a cocycle in $F\left(x_{1}, \ldots, x_{p}, y_{1}, \ldots, y_{p}\right)$. By (iii) (for $i=p$ ) we can write $\tau\left(x_{p+1}\right)$ $=d_{\mathrm{s}}(w)$ for some $w \in F\left(x_{1}, \ldots, x_{p}, y_{1}, \ldots, y_{p}\right)$. In view of (i) this gives

$$
d_{o}\left(x_{p+1}-w\right)=0 \text {. }
$$

Write $w=u+v, u \in\left(x_{1}, \ldots, x_{p}\right) \cdot F\left(x_{1}, \ldots, x_{p}, y_{1}, \ldots, y_{p}\right), v \in$ $F\left(y_{1}, \ldots, y_{p}\right)$.

We prove (iv) by showing that $v=0$. If $v \neq 0$ then for some $q$,

$$
v=\sum_{k=0}^{m} y_{q}^{k} b_{k}
$$

where $b_{k} \in F\left(y_{1}, \ldots, y_{q-1}\right), b_{m} \neq 0$ and $m \geqslant 1$. Suppose this is the case.

Let $I=\left(x_{1}, \ldots, x_{p}\right) \cdot F\left(x_{1}, \ldots, x_{p}, y_{1}, \ldots, y_{p}\right)$ and $\operatorname{set} J=\left(x_{1}, \ldots\right.$, $\left.x_{q-1}\right) \cdot F\left(x_{1}, \ldots, x_{p}, y_{1}, \ldots, y_{p}\right)$. (If $q=1$ set $J=0$.) Then we have the short exact sequence

$$
\begin{aligned}
0 & \rightarrow I \cdot I+J \rightarrow F\left(x_{1}, \ldots, x_{p}, y_{1}, \ldots, y_{p}\right) \\
& \stackrel{\Pi}{\rightarrow}\left[\Gamma \oplus\left(x_{q}, \ldots, x_{n}\right)\right] \otimes F\left(y_{1}, \ldots, y_{p}\right) \rightarrow 0 .
\end{aligned}
$$

It follows from (ii) that $\sigma\left(y_{i}\right) \in I(i \leqslant p)$. The minimality of $\left(\tau ; x_{1}, \ldots\right.$, $x_{n}$ ) implies that $\sigma\left(x_{i}\right)=\tau\left(x_{i}\right) \in I \cdot I(i \leqslant p+1)$. Hence $d_{\sigma}(I) \subset I \cdot I$ and $d_{o}\left(x_{p+1}\right) \in I \cdot I$. Thus applying $\Pi$ to equation (12) we find that 


$$
\Pi d_{\sigma} v=\Pi d_{\sigma}\left(x_{p+1}\right)-\Pi d_{\sigma} u=0 .
$$

Moreover it follows from (ii) that $d_{\alpha} y_{i}=\sigma\left(y_{i}\right) \in J(i<q)$ and $d_{\alpha} y_{q}-x_{q}$ $\in J$. Hence

$$
\Pi d_{\sigma} v=\sum_{k=1}^{m} k \Pi\left(x_{q} y_{q}^{k-1} b_{k}\right)=\sum_{k=1}^{m} k x_{q} y_{q}^{k-1} b_{k} \neq 0 .
$$

This contradiction shows that $v=0$. The induction is now closed and the proof is complete. Q.E.D.

CoROllary. For each $p\left(\sigma ; x_{1}, \ldots, x_{p-1}, y_{1}, \ldots, y_{p-1}, x_{p}, \ldots, x_{n}\right)$ is a tower, and the induced fibre projection

$$
\Pi:\left(F\left(x_{1}, \ldots, x_{n}, y_{1}, \ldots, y_{p-1}\right), d_{\sigma}\right) \rightarrow\left(F\left(x_{p}, \ldots, x_{n}\right), d_{\bar{\tau}}\right)
$$

induces an isomorphism in cohomology.

LemMa 5. Let $\left(\tau ; x_{1}, \ldots, x_{n}\right)$ be a connected, finite, $c$-finite minimal tower. Let $\left[x_{p}\right] \in H\left(F\left(x_{p}, \ldots, x_{n}\right)\right)$ be the class represented by $x_{p}$. Then for each $p$ $(1 \leqslant p \leqslant n),\left[x_{p}\right]$ is nilpotent.

Proof. Let $\left(\sigma ; x_{1}, \ldots, x_{n}, y_{1}, \ldots, y_{n}\right)$ be the tower of Lemma 4 , and fix p. Part (iv) of Lemma 4 implies that for some $w$ of the form

$$
w=\sum_{i=1}^{p-1} x_{i} u_{i}, \quad u_{i} \in F\left(x_{1}, \ldots, x_{p-1}, y_{1}, \ldots, y_{p-1}\right),
$$

we have $d_{\sigma}\left(x_{p}-w\right)=0$ in $F\left(x_{1}, \ldots, x_{n}, y_{1}, \ldots, y_{p-1}\right)$.

Thus if $\Pi$ is the projection in the corollary to Lemma $4, \Pi\left(x_{p}-w\right)=x_{p}$. Let $\alpha \in H\left(F\left(x_{1}, \ldots, x_{n}, y_{1}, \ldots, y_{p-1}\right), d_{\sigma}\right)$ be the cohomology class represented by $x_{p}-w$. Then $\Pi^{*} \alpha=\left[x_{p}\right]$. Since $\Pi^{*}$ is an isomorphism we need only prove that $\alpha^{k}=0$, for some $k$.

Denote by $\left(X, d_{X}\right)$ the Koszul complex of $\left(\tau ; x_{1}, \ldots, x_{n}\right)$. Since $H(X)$ is finite dimensional there is an integer $k \geqslant 2$ such that $H^{j}(X)=0, j \geqslant k$. Let $C \subset X^{k-1}$ be a subspace such that $X^{k-1}=C \oplus\left(\operatorname{ker} d_{X}\right)^{k-1}$. Define a graded, $d_{X}$-stable ideal $I \subset X$ by $I^{j}=0(j<k-1), I^{k-1}=C, I^{j}=X^{j}$ $(j \geqslant k)$.

Let $A=X / I$ and let $d_{A}$ be the derivation induced by $d_{X}$ in $A$. Then the projection $\rho: X \rightarrow A$ is a homomorphism of graded differential algebras, and $\rho^{*}$ is an isomorphism.

On the other hand, $\left(F\left(x_{1}, \ldots, x_{n}, y_{1}, \ldots, y_{p-1}\right), d_{o}\right)$ is the Koszul complex of the tower $\left(X, d_{X} ; \sigma ; y_{1}, \ldots, y_{p-1}\right)$ over $\left(X, d_{X}\right)$. Thus by Lemma $1, \S 2$ there is a tower $\left(A, d_{A} ; \lambda ; y_{1}, \ldots, y_{p-1}\right)$ such that

$$
(\rho \otimes \iota):\left(X \otimes F\left(y_{1}, \ldots, y_{p-1}\right), d_{\sigma}\right) \rightarrow\left(A \otimes F\left(y_{1}, \ldots, y_{p-1}\right), d_{A}+d_{\lambda}\right)
$$

is a homomorphism of graded differential algebras. Moreover $(\rho \otimes \imath)^{*}$ is an isomorphism. 
But $x_{p}-w \in X^{+} \otimes F\left(y_{1}, \ldots, y_{p-1}\right)$ and so $(\rho \otimes \imath)\left(x_{p}-w\right) \in A^{+} \otimes$ $F\left(y_{1}, \ldots, y_{p-1}\right)$. Since $A^{j}=0, j \geqslant k$ this implies that

$$
(\rho \otimes \imath)\left(x_{p}-w\right)^{k}=\left[(\rho \otimes \imath)\left(x_{p}-w\right)\right]^{k}=0 .
$$

Hence $(\rho \otimes \imath)^{*}\left(\alpha^{k}\right)=0$. Since $(\rho \otimes \imath)^{*}$ is an isomorphism, $\alpha^{k}=0$. Q.E.D.

8. Poincaré duality. A finite dimensional graded algebra $A=\sum_{p=0}^{n} A^{p}$ is said to have formal dimension $n$ if $A^{n} \neq 0$ (and $A^{p}=0, p>n$ ). A Poincaré duality algebra (P.d.a.) is a finite dimensional graded commutative algebra $A=\sum_{p=0}^{n} A^{p}$ such that $\operatorname{dim} A^{n}=1$ and such that multiplication defines nondegenerate bilinear maps $A^{p} \times A^{n-p} \rightarrow A^{n}(\cong \Gamma), p=0,1, \ldots$ If $\varepsilon$ is a nonzero element in $A^{n}$ then the scalar product $\langle$,$\rangle in A$ given by

$$
\langle\alpha, \beta\rangle=0, \quad \operatorname{deg} \alpha+\operatorname{deg} \beta \neq n,\langle\alpha, \beta\rangle \varepsilon=\alpha \cdot \beta, \quad \operatorname{deg} \alpha+\operatorname{deg} \beta=n
$$

induces isomorphisms $A^{n-p} \cong\left(A^{p}\right)^{*}$. These are called, respectively, the Poincaré scalar product and the Poincaré isomorphism.

The tensor product of two graded commutative algebras is a P.d.a. if and only if each factor is a P.d.a. If $\left(A, d_{A}\right)$ is a graded differential algebra such that $A$ and $H(A)$ both have formal dimension $n$, and if $A$ is a P.d.a., then so is $H(A)$.

In this section we establish

TheOREM 3. Let $\left(\tau ; x_{1}, \ldots, x_{n}\right)$ be a connected, finite, $c$-finite minimal tower with odd spectral sequence $\left(E_{i}, d_{i}\right)$. Then

(i) $H\left(F\left(x_{1}, \ldots, x_{n}\right), d_{\tau}\right)$ and each $E_{i}(i \geqslant 1)$ have the same formal dimension $m$, given by

$$
m=r-\sum_{i=1}^{n}(-1)^{\operatorname{deg} x_{i}} \operatorname{deg} x_{i},
$$

where $r$ is the number of $x_{i}$ of even degree.

(ii) $H\left(F\left(x_{1}, \ldots, x_{n}\right), d_{\tau}\right)$ and each $E_{i}$ are P.d.a.'s.

(iii) For $i \geqslant 1 E_{i}^{*, q}=0, q<\chi_{\Pi}$ and $E_{i}^{*, q} \neq 0, q=\chi_{\Pi}$.

Exactly as in $\S 1$ (Theorem $1 \Rightarrow$ Theorem $1^{\prime}$ ), Theorem 3 yields

THEOREM $3^{\prime}$. Let $X$ be a connected topological space such that $H^{*}(X ; \mathbf{Q})$ and $\Pi_{\psi}^{*}(X)$ are finite dimensional. Then $H^{*}(X ; \mathbf{Q})$ is a P.d.a. of formal dimension m given by

$$
m=\sum_{i} \operatorname{dim} \Pi_{\psi}^{2 i}(X)-\sum_{k}(-1)^{k} k \operatorname{dim} \Pi_{\psi}^{k}(X) .
$$

If $X$ is simply connected the theorem remains true if $\Pi_{\psi}^{*}(X)$ is replaced by $\Pi_{*}(X) \otimes \mathbf{Q}$ everywhere in the statement.

LEMma 6. Theorem 3 is correct for pure towers. 
Proof. Suppose $\left(\tau ; x_{1}, \ldots, x_{n}\right)$ is a pure tower satisfying the hypotheses of the theorem. Write $R=\left(x_{1}, \ldots, x_{n}\right) ; F(R)=\bigvee Q \otimes \wedge P$. Let $z_{1}, \ldots, z_{r}$ be a homogeneous basis of $Q$ and choose an integer $k \geqslant 2$ so that $z_{i}^{k}=d_{\tau} w_{i}, i=1, \ldots, r$.

Let $U=\left(u_{1}, \ldots, u_{r}\right)$ be a graded vector space with $\operatorname{deg} u_{i}=k \operatorname{deg} z_{i}-1$. Define a graded differential algebra $(\vee Q \otimes \wedge P \otimes \wedge U, d)$ by

$$
d \Phi=d_{\tau} \Phi, \quad \Phi \in \bigvee Q \otimes \wedge P \text { and } d u_{i}=z_{i}^{k}, \quad i=1, \ldots, r .
$$

Then an isomorphism $\phi:\left(\bigvee Q \otimes \wedge P \otimes \wedge U, d_{\tau} \otimes \iota\right) \rightarrow \cong(\vee Q \otimes \wedge P$ $\otimes \wedge U, d)$ is given by

$$
\phi \Phi=\Phi, \quad \Phi \in \bigvee Q \otimes \wedge P, \quad \phi u_{i}=u_{i}-w_{i}, \quad i=1, \ldots, r .
$$

This yields an isomorphism of graded algebras

$$
H\left(\bigvee Q \otimes \wedge P, d_{\tau}\right) \otimes \wedge U \stackrel{\sim}{\rightarrow} H(\bigvee Q \otimes \wedge P \otimes \wedge U, d)
$$

On the other hand, let $A_{i}$ be the truncated polynomial algebra $\bigvee\left(z_{i}\right) / z_{i}^{k}$ and set $A=A_{1} \otimes \cdots \otimes A_{r}$. The projection $\Pi: \bigvee Q \rightarrow A$ determines a graded differential algebra $(A \otimes \wedge P, \bar{d})$.

Moreover, $\Pi$ extends to the homomorphism $\Pi:(\vee Q \otimes \wedge P \otimes \wedge U, d)$ $\rightarrow(A \otimes \wedge P, \bar{d})$ of graded differential algebras given by $\Pi(y)=y, y \in P$ and $\Pi(u)=0, u \in U$. Since the restriction of $\Pi$ to $\vee Q \otimes \wedge U$ induces an isomorphism $H(\bigvee Q \otimes \wedge U) \rightarrow \cong A$, Lemma $1, \S 2$ shows that $\Pi$ induces an isomorphism of graded algebras

$$
\Pi^{*}: H(\vee Q \otimes \wedge P \otimes \wedge U, d) \stackrel{\approx}{\rightarrow} H(A \otimes \wedge P, \bar{d}) \text {. }
$$

Now $A$ and $\wedge P$ are obviously P.d.a.'s of formal dimensions $a$ and $d$ given by

$$
a=\sum_{i=1}^{r}(k-1) \operatorname{deg} z_{i} \text { and } d=\sum_{i=1}^{s} \operatorname{deg} y_{i}
$$

where $y_{1}, \ldots, y_{s}$ is any homogeneous basis of $P$. Hence $A \otimes \wedge P$ is a P.d.a. of formal dimension $a+d$, and $(A \otimes \wedge P)^{a+d}=A^{a} \otimes \bigwedge^{s} P$.

Since $\operatorname{Im} \bar{d} \subset \Sigma_{j<s} A \otimes \bigwedge^{j} P$ it follows that the elements in $A^{a} \otimes \wedge^{s} P$ are not coboundaries; hence $H(A \otimes \wedge P)$ is a P.d.a. of formal dimension $a+d$. Now the isomorphisms (13) and (14) show that $H\left(\bigvee Q \otimes \wedge P, d_{\tau}\right)$ is a P.d.a. of formal dimension

$$
m=a+d-\sum_{i=1}^{r} \operatorname{deg} u_{i}
$$

A simple calculation shows now that $m$ is given by the formula of Theorem 3(i). 
This proves parts (i) and (ii) of Theorem 3 for pure towers. (The odd spectral sequence collapses in this case!) Part (iii) follows at once from formula (3) of Theorem 2. Q.E.D.

LEMMA 7. Let $\left(\tau ; x_{1}, \ldots, x_{n}\right)$ be a tower satisfying the hypotheses of Theorem 3. Then $H\left(F\left(x_{1}, \ldots, x_{n}\right), d_{\tau}\right)$ has formal dimension $m$, where $m$ is given by Theorem 3(i).

Proof. By induction on $n$. For $n=1, \tau=0, x_{1}$ has odd degree and the lemma is trivial. Assume it holds for $n-1$ and distinguish two cases:

Case 1. $\operatorname{deg} x_{1}$ is odd. Write $F\left(x_{1}, \ldots, x_{n}\right)=\wedge x_{1} \otimes F\left(x_{2}, \ldots, x_{n}\right)$ and filter by the ideals $I^{p}=\sum_{j \geqslant p}\left(\bigwedge x_{1}\right)^{j} \otimes F\left(x_{2}, \ldots, x_{n}\right)$. The resulting spectral sequence $\check{E}_{i}$ satisfies (if $\operatorname{deg} x_{1}>1$ )

$$
\check{E}_{2}^{p, q}=\left(\bigwedge x_{1}\right)^{p} \otimes H^{q}\left(F\left(x_{2}, \ldots, x_{n}\right), d_{\bar{\tau}}\right) .
$$

According to the corollary to Proposition $1, \S 6$, the tower $\left(\bar{\tau} ; x_{2}, \ldots, x_{n}\right)$ also satisfies the hypotheses of Theorem 3 . Thus by the induction hypothesis $H\left(F\left(x_{2}, \ldots, x_{n}\right)\right)$ has formal dimension

$$
l=r-\sum_{i=2}^{n}(-1)^{\operatorname{deg} x_{i}} \operatorname{deg} x_{i}=m-\operatorname{deg} x_{1} .
$$

The formal dimension of $\bigwedge x_{1}$ is simply deg $x_{1}=m-l$. Hence $\check{E}_{2}^{p, q}=0$ if $p>m-l$ or $q>l$, while $\check{E}_{2}^{m-l, l} \neq 0$. It follows that $\check{E}_{\infty}^{p, q}=0$ if $p>m-l$ or $q>l$ and $\check{E}_{\infty}^{m-l, l} \neq 0$. Hence $\check{E}_{\infty}$, and so $H\left(F\left(x_{1}, \ldots, x_{n}\right), d_{\tau}\right)$ have formal dimension $m$. The case $\operatorname{deg} x_{1}=1$ is left to the reader.

Case 2. $\operatorname{deg} x_{1}$ is even. Choose $k$ so that $x_{1}^{k}=d_{\tau} w$ and let $U=(u)$ be a 1-dimensional graded space with $\operatorname{deg} u=k \operatorname{deg} x_{1}-1$. Let $A$ be the truncated polynomial algebra $\bigvee\left(x_{1}\right) / x_{1}^{k}$.

The projection $\bigvee\left(x_{1}\right) \rightarrow A$ defines a tower $\left(A, 0 ; \rho, x_{2}, \ldots, x_{n}\right)$. Moreover a slight modification of the proof of Lemma 6 yields an isomorphism of graded algebras:

$$
H\left(F\left(x_{1}, \ldots, x_{n}\right), d_{\tau}\right) \otimes \wedge(u) \cong H\left(A \otimes F\left(x_{2}, \ldots, x_{n}\right), d_{\rho}\right) .
$$

Now filter $A \otimes F\left(x_{2}, \ldots, x_{n}\right)$ and repeat the argument of Case 1 (with $A$ replacing $\left.\wedge\left(x_{1}\right)\right)$ to complete the proof. Q.E.D.

Proof of TheOREM 3. (i) Let $\left(\sigma ; x_{1}, \ldots, x_{n}\right)$ be the associated pure tower; according to Proposition $1, \S 6$ it is $c$-finite. Hence Lemma 6 applies and shows that $H\left(F\left(x_{1}, \ldots, x_{n}\right), d_{\sigma}\right)$ is a P.d.a. of formal dimension $m$. This is therefore true of $E_{1}$ as well (cf. §5).

On the other hand by Lemma $7, H\left(F\left(x_{1}, \ldots, x_{n}\right), d_{\tau}\right)$ also has formal dimension $m$. Since for each $i$

formal $\operatorname{dim} E_{1} \geqslant$ formal $\operatorname{dim} E_{i} \geqslant$ formal $\operatorname{dim} H\left(F\left(x_{1}, \ldots, x_{n}\right), d_{\tau}\right)$, 
it follows that all the $E_{i}$ have formal dimension $m$.

(ii) By Lemma 6, $E_{1}$ is a P.d.a. Since $E_{i}=H\left(E_{i-1}\right)$ and since $E_{i}$ and $E_{i-1}$ have the same formal dimension, an inductive argument shows that each $E_{i}$ is a P.d.a. Hence $E_{\infty}$ is a P.d.a. and so $H\left(F\left(x_{1}, \ldots, x_{n}\right), d_{\tau}\right)$ is a P.d.a.

(iii) The statement $E_{i}^{*, q}=0, q<\chi_{\Pi}$, is Corollary 1 to Theorem $1, \S 6$. Let $q=\chi_{\Pi}$. Then $E_{1}^{*, q}\left(=H_{-q}\left(\bigvee Q \otimes \wedge P, d_{\sigma}\right)\right)$ is a nonzero ideal in $E_{1}$ (cf. Theorem 2, §3). Since $E_{1}$ is a P.d.a. of formal dimension $m$ (cf. Lemma 6 ) we have $E_{1}^{(m)} \subset E_{1}^{*, q}$. It follows that if $i \geqslant 1,0 \neq E_{i}^{(m)} \subset E_{i}^{*, q}$. Q.E.D.

9. Hyperbolic towers. Suppose $A$ is a P.d.a. of even formal dimension $2 m$. Then the Poincare scalar product restricts to a symmetric inner product in the subspace $\Sigma_{j} A^{2 j}$. An inner product space $(X,\langle\rangle$,$) is called hyperbolic if there$ is a subspace $Y$ such that $\left\langle y_{1}, y_{2}\right\rangle=0\left(y_{i} \in Y\right)$ (then $Y$ is called isotropic) and such that $\operatorname{dim} X=2 \operatorname{dim} Y$. If $\sum_{j} A^{2 j}$ is hyperbolic we say $A$ is a hyperbolic P.d.a. Note that this is independent of the choice of basis vector in $A^{2 m}$. In this section we prove

THEOREM 4. Let $\left(\tau ; x_{1}, \ldots, x_{n}\right)$ be a connected, finite, $c$-finite, minimal tower such that $H\left(F\left(x_{1}, \ldots, x_{n}\right), d_{\tau}\right)$ has formal dimension $2 m$.

Assume $\chi_{\Pi}<0$. Then $H\left(F\left(x_{1}, \ldots, x_{n}\right), d_{\tau}\right)$ is a hyperbolic P.d.a. In particular (if $\Gamma \subset \mathbf{R})$ the inner product space $\sum_{j} H^{2 j}\left(F\left(x_{1}, \ldots, x_{n}\right), d_{\tau}\right)$ has zero signature.

COROLlaRY. Let $M$ be a simply connected, compact oriented $4 k$-manifold such that $\Pi_{*}(M ; \mathbf{Q})$ is finite dimensional. Assume that $H^{j}(M ; \mathbf{Q}) \neq 0$ for some odd $j$. Then $\operatorname{sign}(M)=0$.

Proof. It follows from Theorem $1^{\prime}$ that $\chi_{\Pi}<0$. Now apply Theorem 4.

Proof of Theorem 4. In the next section we show (Proposition 3 ) that the theorem holds for pure towers. Hence it holds for the $E_{1}$-term of the odd spectral sequence. On the other hand since $E_{i}$ and $H\left(E_{i}\right)$ have the same formal dimension $2 m$ it follows that there is an isometry $\Sigma_{j} E_{i}^{(2 j)} \cong \Sigma_{j} H^{2 j}\left(E_{i}\right)$ $\oplus X$ where $X$ is a hyperbolic inner product space and $\oplus$ means orthogonal direct sum. If $\sum_{j} E_{i}^{(2 j)}$ is hyperbolic this implies that $\sum_{j} H^{2 j}\left(E_{i}\right)$ is hyperbolic.

Thus an induction argument shows that $\Sigma_{j} E_{\infty}^{(2 j)}$ is hyperbolic; the same must then be true for $\sum_{j} H^{2 j}\left(F\left(x_{1}, \ldots, x_{n}\right), d_{\tau}\right)$. Q.E.D.

REMARK. Theorem 4 shows that the only "interesting" inner products arise when $\operatorname{dim} P=\operatorname{dim} Q$. In this case (cf. Theorem 5, §11) the Koszul complex is the Koszul complex of a pure tower, totally determined by a linear map $\sigma$ : $P \rightarrow \bigvee Q$.

It would be interesting and useful to have an explicit means of calculating invariants of the inner product (e.g. signature) directly from $\sigma$. 
10. The pure case. In this section we prove

Proposition 3. Let $\left(\bigvee Q \otimes \wedge P, d_{\sigma}\right)$ be the Koszul complex of a connected finite, c-finite, pure tower. Suppose $H(\bigvee Q \otimes \wedge P$ ) has formal dimension $2 m$, and assume $\operatorname{dim} P>\operatorname{dim} Q$. Then $\Sigma_{j} H^{2 j}(\bigvee Q \otimes \wedge P)$ is hyperbolic.

LEMma 8. There is a basis $u_{1}, \ldots, u_{s}$ of $P$ (not necessarily homogeneous) with the following properties: Let $I_{i} \subset \vee Q$ be the ideal generated by $\sigma\left(u_{1}\right), \ldots, \sigma\left(u_{i}\right)$. Let $I_{0}=0$. Then

(i) $\overline{\sigma\left(u_{i}\right)} \in \bigvee Q / I_{i-1}$ is not a zero divisor, $1 \leqslant i \leqslant r$, where $r=\operatorname{dim} Q$.

(ii) $\operatorname{dim} \bigvee Q / I_{r}<\infty$.

Proof. We construct $u_{k}(1 \leqslant k \leqslant r)$ by induction on $k$ and extend to any basis of $P$. If $k=1$, let $u_{1}$ be any nonzero element of $P$. Now suppose (for some $k \leqslant r) u_{1}, \ldots, u_{k}$ are constructed, and that (i) holds for $i \leqslant k$.

By the Noether decomposition theorem $I_{k}$ is the finite irredundant intersection of primary ideals in $\bigvee Q$; denote the associated prime ideals by $J_{1}, \ldots$, $J_{l}$ (cf. [6, Chapter 4]). Let $d\left(J_{i}\right)$ be the transcendence degree of $\bigvee Q / J_{i}$.

Suppose $J_{i}$ is not contained in any $J_{j}$. Then according to $[7$, p. 394, Appendix 6], $J_{i}$ has height $k$. Hence by [7, Theorem 20, Chapter 7], $d\left(J_{i}\right)=r$ - $k$. Thus Macaulay's theorem [7, Theorem 26, Chapter 7] applies and asserts that $I_{k}$ is unmixed; i.e., $d\left(J_{i}\right)=r-k, i=1, \ldots, l$. We now distinguish two cases:

Case 1. For some element $u_{k+1}$ of $P, \overline{\sigma\left(u_{k+1}\right)} \in \bigvee Q / I_{k}$ is not a zero divisor. In this case we have constructed a sequence $u_{1}, \ldots, u_{k+1}$ satisfying (i); repeating the argument above yields ideals $J$ with $d(J)=r-k-1$, and so $r \geqslant k+1$.

Case 2. Every $u \in P$ yields a zero divisor $\overline{\sigma(u)}$ in $\bigvee Q / I_{k}$. Choose an infinite sequence $w_{1}, w_{2}, \ldots$ of elements in $P$ such that any subsequence of length $s$ is a basis (possible because char $\Gamma=0$ and so $\Gamma$ is infinite). Each $\overline{\sigma\left(w_{i}\right)}$ is a zero divisor in $\bigvee Q / I_{k}$. Hence by [6, Theorem 11 , Chapter 4] $\sigma\left(w_{i}\right) \in \cup_{j} J_{j}$. By renumbering the $J_{j}$ we can arrange that infinitely many $\sigma\left(w_{i}\right) \in J_{1}$.

It follows that $J_{1}$ contains $\sigma(P)$ and so $\bigvee Q \cdot \sigma(P) \subset J_{1}$. Thus

$\operatorname{dim} \bigvee Q / J_{1} \leqslant \operatorname{dim} \bigvee Q / \bigvee Q \cdot \sigma(P) \leqslant \operatorname{dim} H(\bigvee Q \otimes \wedge P)<\infty$

It follows that $d\left(J_{1}\right)=0$ and so $k=r$.

Thus $u_{1}, \ldots, u_{r}$ are constructed. Moreover, $d\left(J_{j}\right)=0, j=1, \ldots, l$, and so $\operatorname{dim} \bigvee Q / J_{j}<\infty, j=1, \ldots, l$. This implies that $\operatorname{dim} \bigvee Q / I_{r}<\infty$ Q.E.D.

Now define differential algebras $\left(A_{p, t}, d\right), p \leqslant t \leqslant s$, by $\left(A_{p, p}, d\right)=$ $\left(\bigvee Q / I_{p}, 0\right)$ and 


$$
\begin{gathered}
A_{p, t}=\left(\bigvee Q / I_{p}\right) \otimes \wedge\left(u_{p+1}, \ldots, u_{t}\right), \\
d\left(\Phi \otimes u_{\alpha_{0}} \wedge \cdots \wedge u_{\alpha_{q}}\right) \\
=\sum_{j=0}^{q}(-1)^{j} \Phi \cdot \overline{\sigma\left(u_{\alpha_{j}}\right)} \otimes u_{\alpha_{0}} \wedge \cdots \wedge \hat{u}_{\alpha_{j}} \wedge \cdots \wedge u_{\alpha_{q}} \\
\quad 1 \leqslant p \leqslant r .
\end{gathered}
$$

Note that these are not graded differential algebras. Lemma 8 has the following corollary.

COROLLARY. There is an isomorphism of algebras

$$
H\left(\bigvee Q \otimes \wedge P, d_{o}\right) \stackrel{\approx}{\rightarrow} H\left(\bigvee Q / I_{r} \otimes \wedge\left(u_{r+1}, \ldots, u_{s}\right)\right)
$$

which restricts to isomorphisms

$$
H_{k}(\bigvee Q \otimes \wedge P) \stackrel{\cong}{\rightarrow} H_{k}\left(\bigvee Q / I_{r} \otimes \wedge\left(u_{r+1}, \ldots, u_{s}\right)\right)
$$

PROOF. Extend the projections $\Pi: \bigvee Q / I_{p} \rightarrow \bigvee Q / I_{p+1}$ to homomorphisms $\Pi: \bigvee Q / I_{p} \otimes \wedge\left(u_{p+1}\right) \rightarrow \bigvee Q / I_{p+1}$ by setting $\Pi\left(u_{p+1}\right)=$ 0 . It follows directly from Lemma 8 (i) that $\Pi^{*}$ is an isomorphism from

$$
H\left(\bigvee Q / I_{p} \otimes \wedge\left(u_{p+1}\right)\right)
$$

onto $\bigvee Q / I_{p+1}$.

Write $\Pi_{k}=\Pi \otimes \iota: A_{p, p+k} \rightarrow A_{p+1, p+k}$. Assume by induction on $k$ that $\Pi_{k}^{*}$ is an isomorphism. Write $\Pi_{k+1}$ in the form

$$
\Pi_{k+1}=\Pi_{k} \otimes \iota: A_{p, p+k} \otimes \wedge\left(u_{p+k+1}\right) \rightarrow A_{p+1, p+k} \otimes \wedge\left(u_{p+k+1}\right) .
$$

Both sides have a Gysin sequence (cf. the example in \$2) and so the 5-lemma implies that $\Pi_{k+1}^{*}$ is an isomorphism.

In this way we obtain a sequence of isomorphisms

$$
\begin{aligned}
H\left(\bigvee Q / I_{p} \otimes \wedge\left(u_{p+1}, \ldots, u_{s}\right)\right) \stackrel{\sim}{\rightarrow} H\left(\bigvee Q / I_{p+1} \otimes \wedge\left(u_{p+2}, \ldots, u_{s}\right)\right), \\
0 \leqslant p<r .
\end{aligned}
$$

Composing them gives the desired isomorphism. Q.E.D.

LEMMA 9. There is an ideal $I \subset V^{+} Q$ and a basis (not necessarily homogeneous) $u_{1}, \ldots, u_{s}$ of $P$ with the following properties: Let $\rho\left(u_{i}\right) \in \bigvee Q / I$ be the image of $\sigma\left(u_{i}\right)$ under the projection $\bigvee Q \rightarrow \bigvee Q / I$. Then

(i) $\bigvee Q / I$ has finite dimension and the elements in $\bigvee^{+} Q / I$ are nilpotent.

(ii) There is an isomorphism of algebras,

$$
\Psi: H\left(\bigvee Q \otimes \wedge P, d_{\sigma}\right) \cong H\left(\bigvee Q / I \otimes \wedge\left(u_{r+1}, \ldots, u_{s}\right), d_{\rho}\right)
$$

which restricts to isomorphisms 


$$
H_{k}(\bigvee Q \otimes \wedge P) \cong H_{k}\left(\bigvee Q / I \otimes \wedge\left(u_{r+1}, \ldots, u_{s}\right)\right)
$$

PROOF. Let $u_{1}, \ldots, u_{s}$ be the basis of Lemma 8 , and write $B=\bigvee Q / I_{r}$. Multiplication by $\overline{\sigma\left(u_{i}\right)}$ is a linear transformation $\phi_{i}$ of the finite dimensional commutative algebra $B$; in particular $\phi_{i}$ commutes with multiplication by elements of $B$. Let $d=\operatorname{dim} B$.

Then ideals $K_{r+1}, \ldots, K_{s}, L_{r+1}, \ldots, L_{s}$ are defined by the equations:

$$
K_{r+1}=\phi_{r+1}^{d}(B), \quad \phi_{r+1}^{d}\left(L_{r+1}\right)=0, \quad B=K_{r+1} \oplus L_{r+1},
$$

and for $i>r+1$,

$$
K_{i}=\phi_{i}^{d}\left(L_{i-1}\right), \quad \phi_{i}^{d}\left(L_{i}\right)=0, \quad L_{i-1}=K_{i} \oplus L_{i} .
$$

$\phi_{i}$ restricts to an automorphism of $K_{i}$ while each $\phi_{i}$ is nilpotent in $L_{s}$. Moreover $B=K_{r+1} \oplus \cdots \oplus K_{s} \oplus L_{s}$.

Now let $I$ be the inverse image of $K_{r+1} \oplus \cdots \oplus K_{s}$ under the canonical projection $\bigvee Q \rightarrow B$. For $z \in Q$ we know that some $z^{k} \in \bigvee Q \cdot \sigma(P)$. It follows that if $\bar{z}$ is the image of $z$ in $B$ then multiplication by $\bar{z}$ is nilpotent in $L_{s}$. Hence $\bar{z}^{j} \in K_{r+1} \oplus \cdots \oplus K_{s}$ for some $j$ and so $z^{j} \in I$. Thus the elements of $V^{+} Q$ determine nilpotent elements in $\vee Q / I$. This implies (clearly) that $I \subset \bigvee^{+} Q$, and (i) is proved.

To prove (ii) we need only show that projection

$$
\bigvee Q / I_{r} \otimes \wedge\left(u_{r+1}, \ldots, u_{s}\right) \rightarrow \bigvee Q / I \otimes \wedge\left(u_{r+1}, \ldots, u_{s}\right)
$$

induces an isomorphism in cohomology. Since $K_{i}, L_{i}$ are ideals, we have

$$
H\left(B \otimes \wedge\left(u_{r+1}, \ldots, u_{s}\right)\right)=\sum_{i=r+1}^{s} H\left(K_{i} \otimes \wedge\left(u_{r+1}, \ldots, u_{s}\right)\right)
$$

$$
\oplus H\left(L_{s} \otimes \wedge\left(u_{r+1}, \ldots, u_{s}\right)\right) \text {. }
$$

Now $\phi_{i}$ is multiplication by a coboundary, hence it induces zero in cohomology. On the other hand each $K_{k}, L_{j}$ is stable under $\phi_{i}$ and $\phi_{i}$ is an isomorphism in $K_{i}$. Hence $\phi_{i}$ induces an isomorphism in

$$
H\left(K_{i} \otimes \wedge\left(u_{r+1}, \ldots, u_{s}\right)\right) \text {. }
$$

This implies that $H\left(K_{i} \otimes \wedge\left(u_{r+1}, \ldots, u_{s}\right)\right)=0, i=r+1, \ldots, s$, and so (ii) follows from formula (15). Q.E.D.

Denote $\bigvee Q / I$ by $A, \bigvee^{+} Q / I$ by $A^{+}$. (But note that $A$ is not graded!) Let $K \subset A$ be the subspace of elements $x$ such that $x \cdot A^{+}=0$. Finally, denote $\left(u_{r+1}, \ldots, u_{s}\right)$ by $U$. Then clearly

$$
K \otimes u_{r+1} \wedge \cdots \wedge u_{s} \subset\left(A \otimes \wedge^{s-r} U\right) \cap \operatorname{ker} d_{\rho} \subset H_{s-r}(A \otimes \wedge U) .
$$

(The last inclusion is an inclusion because $\operatorname{Im} d_{\rho} \subset \Sigma_{j<s-r} A \otimes \wedge^{j} U$.)

CoRollary. The space $K$ satisfies $\operatorname{dim} K=1$. If $0 \neq a \in K$ then the class $\left[a \otimes u_{r+1} \wedge \cdots \wedge u_{s}\right]$ corresponds under the isomorphism $\Psi$ of Lemma 9 to 
an element of $H^{2 m}(\bigvee Q \otimes \wedge P)$. (Recall that $2 m$ is the formal dimension of $H(\bigvee Q \otimes \wedge P)$.

Proof. It follows from Lemma 9(i) that $K \neq 0$. Let $a \in K$. Then $\left(a \otimes u_{r+1}\right.$ $\left.\wedge \cdots \wedge u_{s}\right) \cdot\left(A^{+} \otimes \wedge U+A \otimes \wedge^{+} U\right)=0$. If $\alpha \in H(\bigvee Q \otimes \wedge P)$ is defined by $\Psi(\alpha)=\left[a \otimes u_{r+1} \wedge \cdots \wedge u_{s}\right]$, then this equation implies that $\alpha \cdot H^{+}(\bigvee Q \otimes \wedge P)=0$. But this condition characterizes

$$
H^{2 m}(\bigvee Q \otimes \wedge P) \text {, }
$$

and $\operatorname{dim} H^{2 m}(\bigvee Q \otimes \wedge P)=1$ by Lemma 6, §8. Q.E.D.

Lemma 10. There is a subspace $X \subset \Sigma_{j} H^{2 j}(\bigvee Q \otimes \wedge P)$ with the following properties:

(i) $2 \operatorname{dim} X=\operatorname{dim}\left(\Sigma_{j} H^{2 j}(\bigvee Q \otimes \wedge P)\right)$.

(ii) $X \cdot X \cap H^{2 m}(\bigvee Q \otimes \wedge P)=0$.

Proof. Clearly $\Sigma_{j} H^{2 j}(\bigvee Q \otimes \wedge P)=\Sigma_{j} H_{2 j}(\bigvee Q \otimes \wedge P)$. Thus we need only find a subspace $Z \subset \Sigma_{j} H_{2 j}(A \otimes \wedge U)$ such that $2 \operatorname{dim} Z=$ $\operatorname{dim}\left(\Sigma_{j} H_{2 j}(A \otimes \wedge U)\right)$ and $Z \cdot Z \cap K \otimes \wedge^{s-r} U=0$ (cf. Lemma 9 and its corollary).

Furthermore, since $H^{2 m}(\bigvee Q \otimes \wedge P) \subset H_{s-r}(\bigvee Q \otimes \wedge P)$, it follows that $s-r=2 k$.

Choose a subspace $N \subset A$ so that $A=N \oplus K=N \oplus(a)$ (a, a basis vector for $K)$. Define a bilinear function $\langle\rangle:, A \times A \rightarrow \Gamma$ by $a_{1} a_{2}-$ $\left\langle a_{1}, a_{2}\right\rangle a \in N$. Since the elements of $A^{+}$are nilpotent, and since $a$ is a basis for $K$, it follows easily that this is a nondegenerate inner product in $A$.

Next assign $\wedge U$ the standard Poincare scalar product determined by the basis vector $u_{r+1} \wedge \cdots \wedge u_{s}$ in $\wedge^{s-r} U$ :

$$
\Phi \wedge \Psi-\langle\Phi, \Psi\rangle u_{r+1} \wedge \cdots \wedge u_{s} \in \sum_{j<s-r} \wedge^{\prime} U
$$

These two scalar products define a scalar product $\langle$,$\rangle in A \otimes \wedge U$, for which $\left\langle A \otimes \wedge^{j} U, A \otimes \wedge^{l} U\right\rangle=0$ unless $j+l=s-r$. In particular, $\left\langle\operatorname{Im} d_{\rho}, 1\right\rangle=0$.

A simple calculation shows as well that

$$
\langle\Phi, \Psi\rangle=\langle\Phi \cdot \Psi, 1\rangle, \quad \Phi, \Psi \in A \otimes \wedge U,
$$

whence

$$
\left\langle d_{\rho} \Phi, \Psi\right\rangle+(-1)^{p}\left\langle\Phi, d_{\rho} \Psi\right\rangle=0
$$

$$
\Phi \in A \otimes \wedge^{p} U, \Psi \in A \otimes \wedge U .
$$

Thus the scalar product of two cocycles depends only on their respective cohomology classes, and so a scalar product is induced in $H(A \otimes \wedge U)$. It satisfies 


$$
\langle\alpha, \beta\rangle=\langle\alpha \cdot \beta, 1\rangle, \quad \alpha, \beta \in H(A \otimes \wedge U) .
$$

Moreover $\left\langle H_{j}(A \otimes \wedge U), H_{l}(A \otimes \wedge U)\right\rangle=0$ if $j+l \neq s-r$; since $s-r=2 k$ the spaces $\Sigma_{j} A \otimes \wedge^{2 j} U$ and $\Sigma_{j} H_{2 j}(A \otimes \wedge U)$ are inner product spaces.

Now choose subspaces $C_{j} \subset A \otimes \wedge^{j} U$ such that $C_{j} \oplus d_{\rho}\left(A \otimes \wedge^{j+1} U\right)=$ ker $d_{\rho} \cap\left(A \otimes \wedge^{j} U\right)$. Then the restriction of $\langle$,$\rangle to \Sigma_{j} C_{2 j}$ is nondegenerate and the inner product spaces $\Sigma_{j} C_{2 j}$ and $\Sigma_{j} H_{2 j}(A \otimes \wedge U)$ are isometric. Write

$$
\sum A \otimes \wedge^{2 j} U=\sum_{j} C_{2 j} \oplus\left(\sum_{j} C_{2 j}\right)^{\perp}
$$

The left-hand side is obviously hyperbolic. Moreover, $\Sigma_{j} d_{\rho}\left(A \otimes \wedge^{2 j+1} U\right)$ is an isotropic subspace of $\left(\Sigma_{j} C_{2 j}\right)^{\perp}$ and

$$
2 \operatorname{dim} \sum_{j} d_{\rho}\left(A \otimes \wedge^{2 j+1} U\right)=\operatorname{dim}\left(\sum C_{2 j}\right)^{\perp} .
$$

Hence $\left(\Sigma C_{2 j}\right)^{\perp}$ is hyperbolic.

It follows that $\Sigma_{j} C_{2 j}$ is hyperbolic; hence so is $\Sigma_{j} H_{2 j}(A \otimes \wedge U)$. Choose an isotropic subspace $Z \subset \Sigma_{j} H_{2 j}(A \otimes \wedge U)$ such that $2 \operatorname{dim} Z=$ $\operatorname{dim}\left(\sum_{j} H_{2 j}(A \otimes \wedge U)\right)$. Formula (18) implies that $Z \cdot Z \cap\left(K \otimes u_{r+1}\right.$ $\left.\wedge \cdots \wedge u_{s}\right)=0$. Q.E.D.

Proof of Proposition 3. Let $X$ be the subspace of Lemma 10. Then there is a basis, $\alpha_{1}, \ldots, \alpha_{N}$ of $X$ with the following property: There are linearly independent elements $\beta_{1}, \ldots, \beta_{N}$ in $\Sigma_{j} H^{2 j}(\vee Q \otimes \wedge P)$ such that

(i) $\beta_{i}$ is homogeneous.

(ii) $\alpha_{i}-\beta_{i} \in \Sigma_{j>\left|\beta_{i}\right|} H^{j}(\bigvee Q \otimes \wedge P)\left(\left|\beta_{i}\right|=\operatorname{deg} \beta_{i}\right)$.

Now let $\langle$, $\rangle$ denote the Poincare scalar product in $H(\vee Q \otimes \wedge P)$. Then $\left\langle\beta_{i}, \beta_{j}\right\rangle=0$ if $\left|\beta_{i}\right|+\left|\beta_{j}\right|<2 m$. On the other hand, if $\left|\beta_{i}\right|+\left|\beta_{j}\right|=2 m$ then (ii) implies that $\left\langle\beta_{i}, \beta_{j}\right\rangle \varepsilon=\beta_{i} \cdot \beta_{j}=\alpha_{i} \cdot \alpha_{j}$. Since

$$
X \cdot X \cap H^{2 m}(\vee Q \otimes \wedge P)=0
$$

this equation implies $\alpha_{i} \alpha_{j}=0$; i.e. $\left\langle\beta_{i}, \beta_{j}\right\rangle=0$ if $\left|\beta_{i}\right|+\left|\beta_{j}\right|=2 m$. Thus the $\beta_{j}$ span an isotropic space $Y \subset \Sigma_{j} H^{2 j}(\bigvee Q \otimes \wedge P)$. Since

$$
\operatorname{dim} Y=\operatorname{dim} X=\frac{1}{2} \sum_{j} \operatorname{dim} H^{2 j}(\bigvee Q \otimes \wedge P),
$$

the inner product space $\Sigma_{j} H^{2 j}(\bigvee Q \otimes \wedge P)$ is hyperbolic. Q.E.D.

11. The case that $\chi_{\Pi}=0$. The object of this section is to establish

THEOREM 5. Let $\left(\tau ; x_{1}, \ldots, x_{n}\right)$ be a connected, finite, c-finite, minimal tower. Assume $\chi_{\Pi}=0$. Then the Koszul complex of the tower and the Koszul complex of the associated pure tower are isomorphic as graded differential 
algebras: $\left(\bigvee Q \otimes \wedge P, d_{\tau}\right) \cong\left(\bigvee Q \otimes \wedge P, d_{\sigma}\right)$.

Throughout the section $\left(\tau ; x_{1}, \ldots, x_{n}\right)$ denotes a fixed tower satisfying the hypotheses of the theorem; $R=\left(x_{1}, \ldots, x_{n}\right) ; F(R)=\bigvee Q \otimes \wedge P ;(\sigma ;$ $\left.x_{1}, \ldots, x_{n}\right)$ is the associated pure tower. To establish the theorem we may assume without loss of generality that

$$
\operatorname{deg} x_{1} \leqslant \operatorname{deg} x_{2} \leqslant \ldots
$$

We assume this throughout the section.

Lemma 11. Suppose $x_{i}$ has even degree. Then for some

$$
u_{i} \in F^{+}\left(x_{1}, \ldots, x_{i-1}\right) \cdot F^{+}\left(x_{1}, \ldots, x_{i-1}\right), d_{\tau}\left(x_{i}+u_{i}\right)=0 .
$$

Proof. By Corollary $4, \S 6$, the odd spectral sequence collapses at the $E_{1}$-term. Moreover $d_{0}\left(x_{i}\right)=0$. Thus $x_{i}$ represents an element in $E_{\infty}^{p, 0}(p=$ $\left.\operatorname{deg} x_{i}\right)$. It follows that $d_{\tau}\left(x_{i}+u_{i}\right)=0$ for some $u_{i} \in \sum_{j>0} F(R)^{j+p,-j}$. But $F(R)^{j+p,-j} \subset \bigvee Q \otimes \wedge^{j} P$. Since $u_{i}$ has even degree $p$ this gives

$$
u_{i} \in \sum_{j>2} \bigvee Q \otimes \bigwedge^{j} P \subset F^{+}(R) \cdot F^{+}(R)
$$

It follows now from (19) that $u_{i} \in F^{+}\left(x_{1}, \ldots, x_{i-1}\right) \cdot F^{+}\left(x_{1}, \ldots, x_{i-1}\right)$. Q.E.D.

Now define an automorphism $\phi$ of the graded algebra $F(R)$ by setting $\phi x_{i}=x_{i}\left(x_{i} \in P\right)$ and $\phi x_{i}=x_{i}+u_{i}\left(x_{i} \in Q\right)$. Then $\phi$ restricts to automorphisms of each $F\left(x_{1}, \ldots, x_{i}\right)$. Hence a tower $\left(\rho ; x_{1}, \ldots, x_{n}\right)$ is defined by

$$
\rho\left(x_{i}\right)=\phi^{-1} d_{\tau} \phi\left(x_{i}\right), \quad i=1, \ldots, n .
$$

Lemma 11 yields

$$
\rho(x)=0, \quad x \in Q .
$$

Clearly $\phi:\left(F(R), d_{\rho}\right) \rightarrow \simeq\left(F(R), d_{\tau}\right)$ is an isomorphism of graded differential algebras. It follows (cf. $\S 2)$ that $\left(\rho ; x_{1}, \ldots, x_{n}\right)$ is a connected, finite, $c$-finite, minimal tower, with zero homotopy Euler characteristic. In view of (20) this tower can be rearranged (cf. §2) in the form $\left(\rho ; z_{1}, \ldots, z_{m}, y_{1}, \ldots\right.$, $\left.y_{m}\right)$, where the $z_{i}$ are a basis of $Q$ and the $y_{i}$ a basis of $P .\left(z_{1}, \ldots, y_{m}\right.$ is a permutation of $x_{1}, \ldots, x_{n}$.)

Let $\left(\lambda ; z_{1}, \ldots, z_{m}, y_{1}, \ldots, y_{m}\right)$ be the associated pure tower. Proposition 1 , applied to $\left(\rho ; x_{1}, \ldots, x_{n}\right)$ shows that $H\left(\vee Q \otimes \wedge P, d_{\lambda}\right)$ has finite dimension. Hence Theorem 2 implies that $H_{+}\left(\vee Q \otimes \wedge P, d_{\lambda}\right)=0$. Let $P_{i}$ be the subspace of $P$ spanned by $y_{1}, \ldots, y_{i}$. Lemma $2, \S 3$ implies now that for each $i, H_{+}\left(\vee Q \otimes \wedge P_{i}, d_{\lambda}\right)=0$.

LEMmA 12. For each $i$ the inclusion $\theta: \bigvee Q \rightarrow \bigvee Q \otimes \wedge P_{i}$ induces $a$ surjective homomorphism $\theta^{*}: \bigvee Q \rightarrow H\left(\bigvee Q \otimes \wedge P_{i}, d_{\rho}\right)$. 
Proof. Since $H_{+}\left(\bigvee Q \otimes \wedge P_{i}, d_{\lambda}\right)=0, H\left(\bigvee Q \otimes \wedge P_{i}, d_{\lambda}\right)$ is evenly graded. Thus if $\left(F_{k}, d_{k}\right)$ is the odd spectral sequence for $\left(\rho ; z_{1}, \ldots\right.$, $\left.z_{m}, y_{1}, \ldots, y_{i}\right), F_{1}=H\left(\bigvee Q \otimes \wedge P_{i}, d_{\lambda}\right)$ is evenly graded. Hence $F_{1}=F_{\infty}$.

Now filter $\bigvee Q$ by the ideals $\hat{I}^{p}=\sum_{j \geqslant p}(\bigvee Q)^{j}$. The corresponding spectral sequence is given $\hat{F}_{k}=\bigvee Q, \hat{d}_{k}=0$.

Observe that $\theta$ is filtration preserving and so induces a homomorphism $\theta_{k}$ : $\hat{F}_{k} \rightarrow F_{k}$ of spectral sequences. In particular, $\theta_{1}: \bigvee Q \rightarrow H\left(\bigvee Q \otimes \wedge P_{i}, d_{\lambda}\right)$ is surjective, since $H_{+}\left(\bigvee Q \otimes \wedge P_{i}, d_{\lambda}\right)=0$. But $F_{1}=F_{\infty}, \hat{F}_{1}=\hat{F}_{\infty}$; thus $\theta_{\infty}=\theta_{1}$ and so $\theta_{\infty}$ is surjective. This implies at once that $\theta^{*}$ is surjective. Q.E.D.

Proof of Theorem 5. We continue the notation developed above. Since ( $\rho$; $\left.z_{1}, \ldots, z_{m}, y_{1}, \ldots, y_{m}\right)$ is a tower it follows that

$$
\rho\left(y_{i}\right) \in \operatorname{ker} d_{\rho} \cap\left(\bigvee Q \otimes \wedge P_{i-1}\right), \quad i=1,2, \ldots, m .
$$

In view of Lemma 12 above we can write

$$
\rho\left(y_{i}\right)=v_{i}+d_{\rho} w_{i}, \quad v_{i} \in \bigvee Q, \quad w_{i} \in \bigvee Q \otimes \wedge P_{i-1} .
$$

Define an automorphism $\Psi$ of the graded algebra $\bigvee Q \otimes \wedge P$ by setting

$$
\Psi\left(z_{i}\right)=z_{i}, \quad \Psi\left(y_{i}\right)=y_{i}-w_{i}, \quad i=1, \ldots, m .
$$

Then define $\gamma: R \rightarrow \bigvee Q \otimes \wedge P$ by

$$
\gamma\left(y_{i}\right)=v_{i} \text { and } \gamma\left(z_{i}\right)=0 .
$$

It follows from the definition that $\Psi d_{\gamma}=d_{\rho} \Psi$.

In particular, $\operatorname{Im} \gamma \subset F^{+}(R) \cdot F^{+}(R)$. In view of (21) this implies that $(\gamma$; $\left.x_{1}, \ldots, x_{n}\right)$ is a pure, minimal tower. Thus it coincides with the associated pure tower.

Now consider the isomorphism $\phi \circ \Psi:\left(F(R), d_{\gamma}\right) \rightarrow \widetilde{\simeq}\left(F(R), d_{\tau}\right)$ of Koszul complexes. According to $\$ 5$ it induces an isomorphism of the odd spectral sequences. The isomorphism of the $E_{0}$-terms can be written $\alpha:\left(F(R), d_{\gamma}\right)$ $\rightarrow \approx\left(F(R), d_{\sigma}\right)$. Thus $\phi \circ \psi \circ \alpha^{-1}:\left(F(R), d_{\sigma}\right) \rightarrow \cong\left(F(R), d_{\tau}\right)$ is the desired isomorphism. Q.E.D.

Corollary 1. $H^{*}\left(F(R), d_{\tau}\right) \cong \bigvee Q / \bigvee Q \cdot \sigma(P)$.

Proof. Apply Theorem 2.

Corollary 2. Suppose the bases $y_{i}$ of $P$ and $z_{i}$ of $Q$ satisfy $\operatorname{deg} y_{i}=g_{i}$, $\operatorname{deg} z_{i}=k_{i}$. Then

$$
\sum_{p} \operatorname{dim} H^{p}\left(F(R), d_{\tau}\right) t^{p}=\prod_{i=1}^{m}\left(1-t^{g_{i}+1}\right) \prod_{i=1}^{m}\left(1-t^{k_{i}}\right)^{-1} .
$$

Moreover the Euler characteristic, $\chi_{c}$ (equals the dimension of the cohomology) is given by formula $\chi_{c}=\left(\left(g_{i}+1\right) \ldots\left(g_{m}+1\right)\right) /\left(k_{1} \ldots k_{m}\right)$.

Proof. See [4] or [3, Chapter 2]. 


\section{REFERENCES}

1. C. Allday, On the rank of a space, Trans. Amer. Math. Soc. 166 (1972), 173-185. MR 45 \#1158.

2. H. Cartan, La transgression dans un groupe de Lie et dans un espace fibré principal, Colloque de Topologie (espaces fibrés), Bruxelles (1950), Thone, Liège; Masson, Paris, 1951, pp. 57-71. MR 13, 107.

3. W. H. Greub, S. Halperin and J. R. Vanstone, Connections, curvature and cohomology, vol. III, Academic Press, New.York, 1975.

4. J.-L. Koszul, Sur un type d'algèbres différentielles en rapport avec la transgression, Colloque de Topologie (espaces fibrés), Bruxelles (1950), Thone, Liège; Masson, Paris, 1951, pp. 73-81. MR 13, 109.

5. D. Sullivan, Infinitesimal computations in topology (preprint).

6. O. Zariski and P. Samuel, Commutative algebra, Vol. I, Van Nostrand, Princeton, N.J., 1958. MR 19, 833.

7. ___ Commutative algebra. Vol. II, Van Nostrand, Princeton, N.J., 1960. MR 22 \#11006.

U.E.R. Mathematiques Pures et Appliques, Universite des Sciences et Techiques de Lille I, B. P. 36, 59650 Villeneuve d’AscQ, France

Department of Mathematics, University of Toronto, Toronto, Ontario, M5S 1A7, CANADA 